- Supporting Information -

\title{
Kinetics of the Reactions of Halide Anions with Carbocations: Quantitative Energy Profiles for $\mathrm{S}_{\mathrm{N}} 1$ Reactions
}

\author{
Shinya Minegishi, ${ }^{a}$ Robert Loos, ${ }^{a}$ Shinjiro Kobayashi, ${ }^{b}$ and Herbert Mayr ${ }^{*}, a$ \\ ${ }^{a}$ Department Chemie und Biochemie der Ludwig-Maximilians-Universität München, \\ Butenandtstrasse 5-13 (Haus F), D-81377 München, Germany \\ ${ }^{b}$ IFOC, Kyushu University, Higashi-ku, Fukuoka 812-8581, Japan \\ E-Mail: Herbert.Mayr@cup.uni-muenchen.de
}

Table of contents

Derivation of eq. $5\left(r_{1}+r_{2}=k_{2, \mathrm{X}^{-}}-\left[\mathrm{X}^{-}\right]+k_{\mathrm{SOH}}+k_{\text {ionization }}\right)$

Correlations of $\boldsymbol{N}$ against $\boldsymbol{Y}$ for different solvents

Details of the kinetic experiments:

Reactions with chloride $\left(n \mathrm{Bu}_{4} \mathrm{~N}^{+} \mathrm{Cl}^{-}\right)$

Reactions with bromide $\left(n \mathrm{Bu}_{4} \mathrm{~N}^{+} \mathrm{Br}^{-}\right)$

References 
Derivation of eq. $5\left(r_{1}+r_{2}=k_{2, X^{-}}-\left[\mathrm{X}^{-}\right]+k_{\mathrm{SOH}}+\boldsymbol{k}_{\text {ionization }}\right)^{1,2}$

$$
\begin{aligned}
& \mathrm{A} \frac{k_{1}}{k_{2}} \mathrm{~B} \\
& \mathrm{~A} \stackrel{k_{3}}{\longrightarrow} \mathrm{C}
\end{aligned}
$$

In the parallel two first-order reactions in which one step is reversible (eqs. S1 and S2), the set of differential equations is given by

$$
\begin{aligned}
& d[\mathrm{~A}] / d t=-\left(k_{1}+k_{3}\right)[\mathrm{A}]+k_{2}[\mathrm{~B}] \\
& d[\mathrm{~B}] / d t=k_{1}[\mathrm{~A}]-k_{2}[\mathrm{~B}] \\
& d[\mathrm{C}] / d t=k_{3}[\mathrm{~A}]
\end{aligned}
$$$$
[\mathrm{A}]+[\mathrm{B}]+[\mathrm{C}]=[\mathrm{A}]_{0}
$$

Differentiating eq. S3 with respect to $t$ leads to

$d^{2}[\mathrm{~A}] / \mathrm{d} t^{2}=-\left(k_{1}+k_{3}\right) d[\mathrm{~A}] / d t+k_{2} d[\mathrm{~B}] / d t$

from eqs. S3 and S4

$d[\mathrm{~B}] / d t=-k_{3}[\mathrm{~A}]-d[\mathrm{~A}] / d t$

Elimination of $[\mathrm{B}]$ and $d[\mathrm{~B}] / d t$ from eqs. $\mathrm{S} 7$ and $\mathrm{S} 8$ gives

$d^{2}[\mathrm{~A}] / \mathrm{d} t^{2}+\left(k_{1}+k_{2}+k_{3}\right) d[\mathrm{~A}] / d t+k_{2} k_{3}[\mathrm{~A}]=$

The general solution of eq. S9is ${ }^{3}$

$[A]=A_{1}\left[\exp \left(-r_{1} \mathrm{t}\right)\right]+A_{2}\left[\exp \left(-r_{2} \mathrm{t}\right)\right]$

$\left.r_{1}, r_{2}=1 / 2\left[k_{1}+k_{2}+k_{3} \pm\left\{\left(k_{1}+k_{2}+k_{3}\right)^{\wedge} 2-4 k_{2} k_{3}\right)\right\}^{\wedge} 0.5\right](7.16)$

Therefore:

$$
\begin{aligned}
& r_{1}+r_{2}=k_{1}+k_{2}+k_{3} \\
& \boldsymbol{r}_{\mathbf{1}}+\boldsymbol{r}_{\mathbf{2}}=\boldsymbol{k}_{\mathbf{2}, \mathbf{X}}-\left[\mathbf{X}^{-}\right]+\boldsymbol{k}_{\mathrm{SOH}}+\boldsymbol{k}_{\text {ionization }}
\end{aligned}
$$




\section{Correlations of $\boldsymbol{N}$ against $\boldsymbol{Y}$ for different solvents}

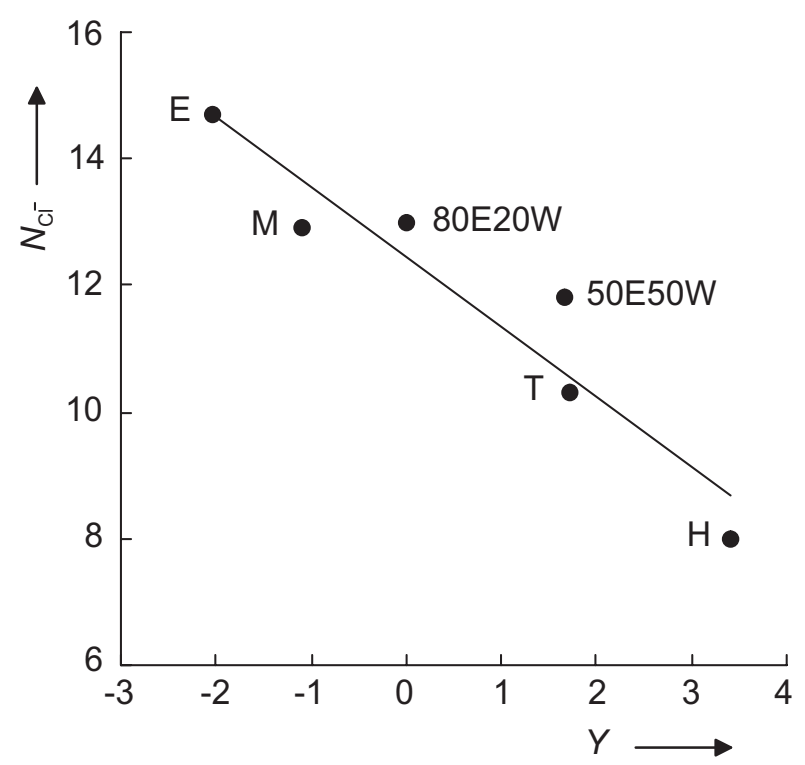

Figure S1 Correlation of $N$ parameters of $\mathrm{Cl}^{-}$in several solvents with solvent ionizing power $Y^{4}$ (derived from $t$-butyl chloride solvolysis) of the solvents. $N=-1.11 Y+12.5, n=6, R^{2}=0.898$.

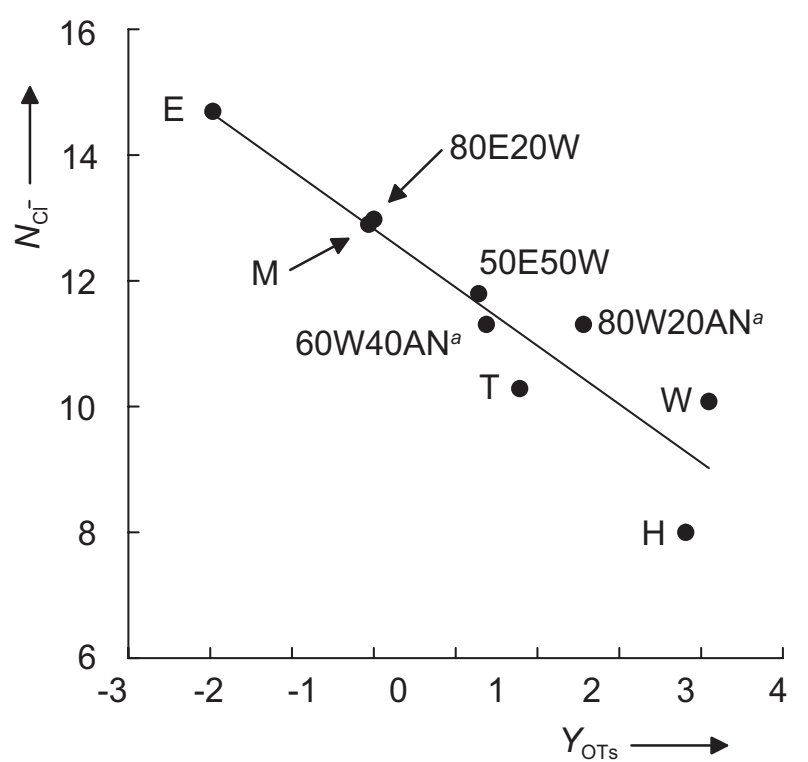

Figure S2 Correlation of $N$ parameters of $\mathrm{Cl}^{-}$in several solvents with solvent ionizing power $Y_{\mathrm{OTs}}{ }^{4}$ (derived from 1- or 2-adamantyl tosylates solvolyses) of the solvents. ${ }^{a}$ Interpolated values from ref $4 . N$ $=-0.93 Y_{\mathrm{OTs}}+12.8, n=9, R^{2}=0.854$. 


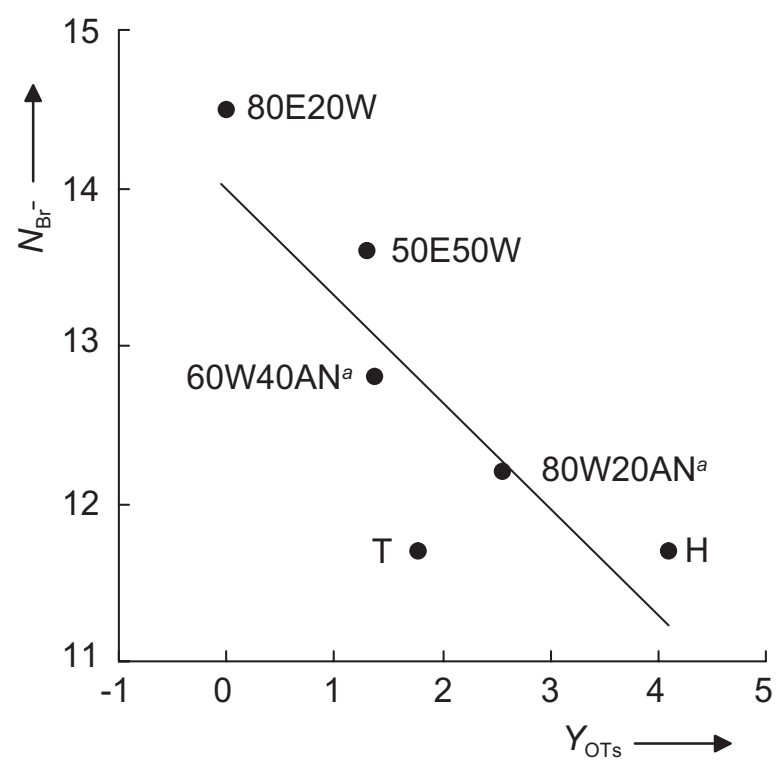

Figure S3 Correlation of $N$ parameters of $\mathrm{Br}^{-}$in several solvents with solvent ionizing power $Y_{\mathrm{OTs}}{ }^{4}$ (derived from 1- or 2-adamantyl tosylates solvolyses) of the solvents. ${ }^{a}$ Interpolated values from ref $4 . N$ $=-0.67 Y_{\mathrm{OTs}}+14.0, n=6, R^{2}=0.683$. 


\section{Details of the kinetic experiments}

\section{Reactions with chloride $\left(n \mathrm{Bu}_{4} \mathrm{~N}^{+} \mathrm{Cl}^{-}\right)$}

Table S1. (ani) ${ }_{2} \mathrm{CH}^{+}$from (ani) $)_{2} \mathrm{CHOAr}+\mathrm{Cl}^{-}$in methanol at $20^{\circ} \mathrm{C}$ (Laser flash, detection at $\left.500 \mathrm{~nm}\right){ }^{a}$

\begin{tabular}{llll}
\hline No. & {$[\text { Precursor }]_{0} / \mathrm{M}$} & {$\left[\mathrm{Cl}^{-}\right] / \mathrm{M}$} & $k_{\text {obs }} / \mathrm{s}^{-1}$ \\
\hline F-63-1 & $1.99 \times 10^{-4}$ & $1.67 \times 10^{-3}$ & $6.34 \times 10^{6}$ \\
F-63-2 & $1.99 \times 10^{-4}$ & $3.33 \times 10^{-3}$ & $6.61 \times 10^{6}$ \\
F-63-3 & $1.99 \times 10^{-4}$ & $5.00 \times 10^{-3}$ & $6.50 \times 10^{6}$ \\
F-63-4 & $1.99 \times 10^{-4}$ & $6.67 \times 10^{-3}$ & $6.72 \times 10^{6}$ \\
F-63-5 & $1.99 \times 10^{-4}$ & $8.34 \times 10^{-3}$ & $6.62 \times 10^{6}$ \\
F-63-6 & $1.99 \times 10^{-4}$ & $1.00 \times 10^{-2}$ & $7.38 \times 10^{6}$ \\
F-63-7 & $1.99 \times 10^{-4}$ & $2.00 \times 10^{-3}$ & $7.40 \times 10^{6}$ \\
F-63-8 & $1.99 \times 10^{-4}$ & $3.00 \times 10^{-3}$ & $7.85 \times 10^{6}$ \\
F-63-9 & $1.99 \times 10^{-4}$ & $3.59 \times 10^{-3}$ & $8.15 \times 10^{6}$ \\
F-63-10 & $1.99 \times 10^{-4}$ & $4.00 \times 10^{-3}$ & $8.59 \times 10^{6}$ \\
F-63-11 & $1.99 \times 10^{-4}$ & $4.28 \times 10^{-3}$ & $8.69 \times 10^{6}$ \\
\hline
\end{tabular}

${ }^{a} \mathrm{OAr}=4$-cyanophenoxide

$k_{2}=5.33 \times 10^{7} \mathrm{M}^{-1} \mathrm{~s}^{-1}$

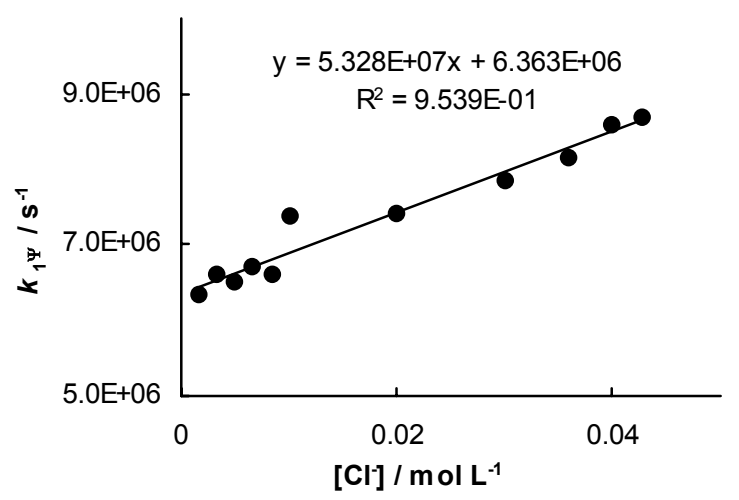


Table S2. (ani) $)_{2} \mathrm{CH}^{+}$from (ani) $)_{2} \mathrm{CHOAr}+\mathrm{Cl}^{-}$in ethanol at $20^{\circ} \mathrm{C}$ (Laser flash, detection at $\left.500 \mathrm{~nm}\right) .{ }^{a}$

\begin{tabular}{llll}
\hline No. & {$[\text { Precursor }]_{0} / \mathrm{M}$} & {$\left[\mathrm{Cl}^{-}\right] / \mathrm{M}$} & $k_{\mathrm{obs}} / \mathrm{s}^{-1}$ \\
\hline F73-1 & $1.99 \times 10^{-4}$ & $8.85 \times 10^{-4}$ & $6.48 \times 10^{6}$ \\
F73-2 & $1.99 \times 10^{-4}$ & $3.01 \times 10^{-3}$ & $8.26 \times 10^{6}$ \\
F73-3 & $1.99 \times 10^{-4}$ & $4.96 \times 10^{-3}$ & $9.74 \times 10^{6}$ \\
F73-4 & $1.99 \times 10^{-4}$ & $7.08 \times 10^{-3}$ & $1.08 \times 10^{7}$ \\
F73-5 & $1.99 \times 10^{-4}$ & $8.85 \times 10^{-3}$ & $1.22 \times 10^{7}$ \\
F73-6 & $1.99 \times 10^{-4}$ & $1.20 \times 10^{-2}$ & $1.22 \times 10^{7}$ \\
F73-7 & $1.99 \times 10^{-4}$ & $1.50 \times 10^{-2}$ & $1.24 \times 10^{7}$ \\
F73-8 & $1.99 \times 10^{-4}$ & $1.77 \times 10^{-2}$ & $1.38 \times 10^{7}$ \\
\hline
\end{tabular}

${ }^{a} \mathrm{OAr}=4$-cyanophenoxide

$k_{2}=6.99 \times 10^{8} \mathrm{M}^{-1} \mathrm{~s}^{-1}$

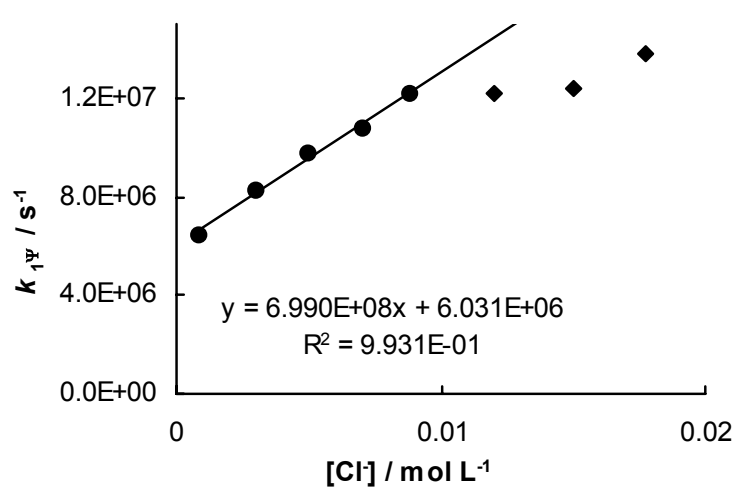


Table S3. (ani) ${ }_{2} \mathrm{CH}^{+}$from (ani) $)_{2} \mathrm{CHOAr}+\mathrm{Cl}^{-}$in $80 \mathrm{E} 20 \mathrm{~W}$ at $20^{\circ} \mathrm{C}$ (Laser flash, detection at $\left.500 \mathrm{~nm}\right){ }^{a}$

\begin{tabular}{llll}
\hline No. & {$[\text { Precursor }]_{0} / \mathrm{M}$} & {$\left[\mathrm{Cl}^{-}\right] / \mathrm{M}$} & $k_{\text {obs }} / \mathrm{s}^{-1}$ \\
\hline F76-1 & $1.99 \times 10^{-4}$ & $5.57 \times 10^{-3}$ & $1.94 \times 10^{6}$ \\
F76-2 & $1.99 \times 10^{-4}$ & $1.05 \times 10^{-2}$ & $2.19 \times 10^{6}$ \\
F76-3 & $1.99 \times 10^{-4}$ & $1.55 \times 10^{-2}$ & $2.52 \times 10^{6}$ \\
F76-4 & $1.99 \times 10^{-4}$ & $2.11 \times 10^{-2}$ & $2.70 \times 10^{6}$ \\
F76-5 & $1.99 \times 10^{-4}$ & $2.60 \times 10^{-2}$ & $2.89 \times 10^{6}$ \\
F76-6 & $1.99 \times 10^{-4}$ & $3.10 \times 10^{-2}$ & $3.44 \times 10^{6}$ \\
F76-7 & $1.99 \times 10^{-4}$ & $3.72 \times 10^{-2}$ & $3.93 \times 10^{6}$ \\
F76-8 & $1.99 \times 10^{-4}$ & $4.34 \times 10^{-2}$ & $3.85 \times 10^{6}$ \\
F76-9 & $1.99 \times 10^{-4}$ & $5.97 \times 10^{-2}$ & $3.82 \times 10^{6}$ \\
F76-10 & $1.99 \times 10^{-4}$ & $8.01 \times 10^{-2}$ & $4.09 \times 10^{6}$ \\
\hline
\end{tabular}

${ }^{a} \mathrm{OAr}=4$-cyanophenoxide

$k_{2}=6.05 \times 10^{7} \mathrm{M}^{-1} \mathrm{~s}^{-1}$

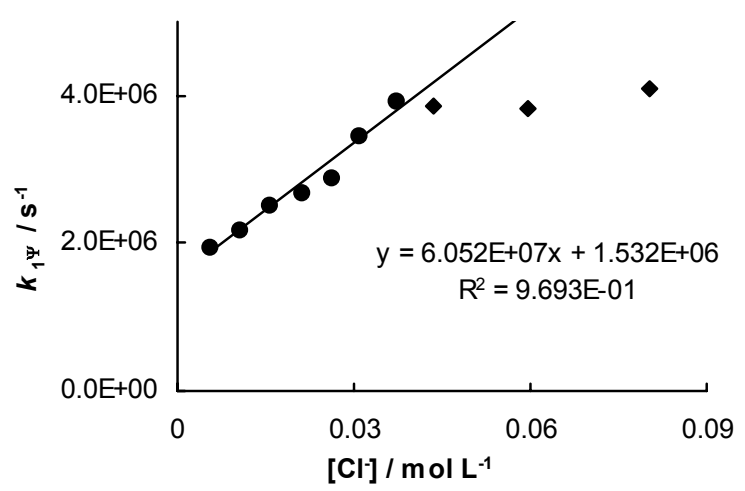


Table S4. (ani) ${ }_{2} \mathrm{CH}^{+}$from (ani) ${ }_{2} \mathrm{CHOAc}+\mathrm{Cl}^{-}$in $20 \mathrm{~W} 80 \mathrm{AN}$ at $20^{\circ} \mathrm{C}$ (Laser flash, detection at $\left.500 \mathrm{~nm}\right){ }^{a}$

\begin{tabular}{llll}
\hline No. & {$[\text { Precursor }]_{0} / \mathrm{M}$} & {$\left[\mathrm{Cl}^{-}\right] / \mathrm{M}$} & $k_{\mathrm{obs}} / \mathrm{s}^{-1}$ \\
\hline F168-1 & $1.59 \times 10^{-4}$ & $2.33 \times 10^{-3}$ & $3.45 \times 10^{5}$ \\
F168-2 & $1.59 \times 10^{-4}$ & $3.26 \times 10^{-3}$ & $4.32 \times 10^{5}$ \\
F168-3 & $1.59 \times 10^{-4}$ & $4.65 \times 10^{-3}$ & $5.53 \times 10^{5}$ \\
F168-4 & $1.59 \times 10^{-4}$ & $6.98 \times 10^{-3}$ & $7.73 \times 10^{5}$ \\
F168-5 & $1.59 \times 10^{-4}$ & $7.91 \times 10^{-3}$ & $9.05 \times 10^{5}$ \\
F168-6 & $1.59 \times 10^{-4}$ & $9.30 \times 10^{-3}$ & $9.92 \times 10^{5}$ \\
F168-7 & $1.59 \times 10^{-4}$ & $1.16 \times 10^{-2}$ & $1.03 \times 10^{6}$ \\
\hline
\end{tabular}

${ }^{a} \mathrm{OAc}=$ acetate

$k_{2}=9.53 \times 10^{7} \mathrm{M}^{-1} \mathrm{~s}^{-1}$

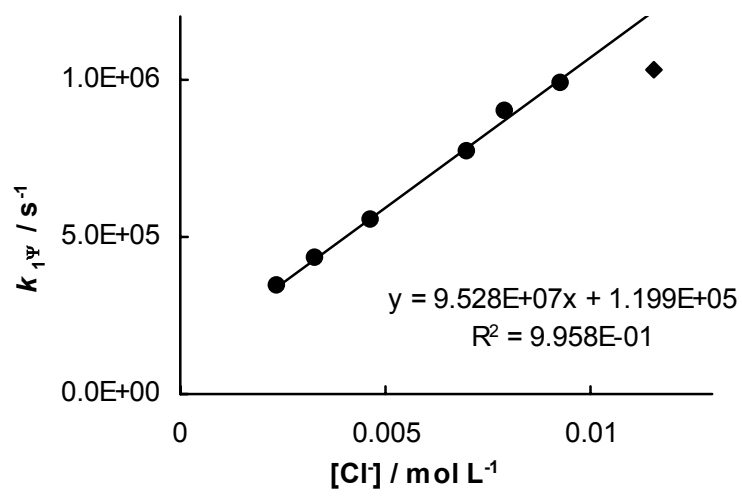


Table S5. (ani) $)_{2} \mathrm{CH}^{+}$from (ani) $)_{2} \mathrm{CHOAc}+\mathrm{Cl}^{-}$in $50 \mathrm{~W} 50 \mathrm{E}$ at $20^{\circ} \mathrm{C}$ (Laser flash, detection at $\left.500 \mathrm{~nm}\right){ }^{a}$

\begin{tabular}{llll}
\hline No. & {$[\text { Precursor }]_{0} / \mathrm{M}$} & {$\left[\mathrm{Cl}^{-}\right] / \mathrm{M}$} & $k_{\mathrm{obs}} / \mathrm{s}^{-1}$ \\
\hline F161-1 & $1.59 \times 10^{-4}$ & $2.03 \times 10^{-3}$ & $4.86 \times 10^{5}$ \\
F161-2 & $1.59 \times 10^{-4}$ & $4.07 \times 10^{-3}$ & $5.34 \times 10^{5}$ \\
F161-3 & $1.59 \times 10^{-4}$ & $6.10 \times 10^{-3}$ & $5.33 \times 10^{5}$ \\
F161-4 & $1.59 \times 10^{-4}$ & $8.14 \times 10^{-3}$ & $5.42 \times 10^{5}$ \\
F161-5 & $1.59 \times 10^{-4}$ & $1.02 \times 10^{-2}$ & $5.99 \times 10^{5}$ \\
F161-6 & $1.59 \times 10^{-4}$ & $1.53 \times 10^{-2}$ & $6.60 \times 10^{5}$ \\
F161-7 & $1.59 \times 10^{-4}$ & $1.86 \times 10^{-2}$ & $6.69 \times 10^{5}$ \\
F161-8 & $1.59 \times 10^{-4}$ & $2.03 \times 10^{-2}$ & $7.25 \times 10^{5}$ \\
F161-9 & $1.59 \times 10^{-4}$ & $2.54 \times 10^{-2}$ & $7.68 \times 10^{5}$ \\
\hline
\end{tabular}

${ }^{a} \mathrm{OAc}=$ acetate

$k_{2}=1.20 \times 10^{7} \mathrm{M}^{-1} \mathrm{~s}^{-1}$

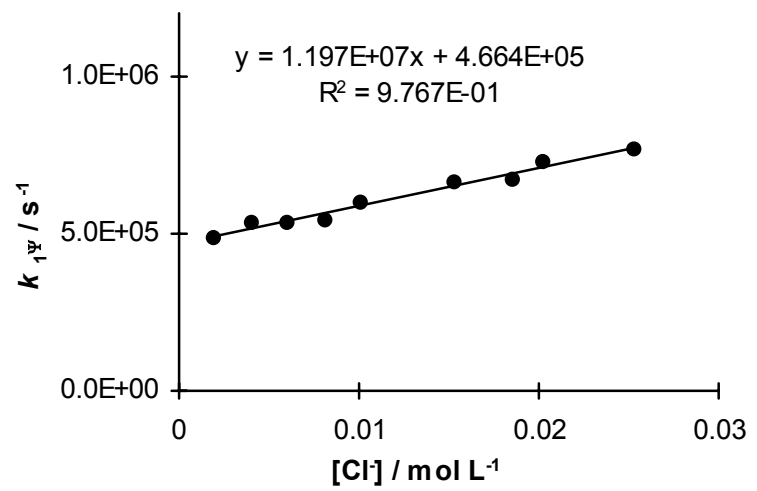


Table S6. (ani) $)_{2} \mathrm{CH}^{+}$from (ani) ${ }_{2} \mathrm{CHOAc}+\mathrm{Cl}^{-}$in $50 \mathrm{M} 50 \mathrm{AN}$ at $20^{\circ} \mathrm{C}$ (Laser flash, detection at $500 \mathrm{~nm}$ ). ${ }^{a}$

\begin{tabular}{llll}
\hline No. & {$[\text { Precursor }]_{0} / \mathrm{M}$} & {$\left[\mathrm{Cl}^{-}\right] / \mathrm{M}$} & $k_{\mathrm{obs}} / \mathrm{s}^{-1}$ \\
\hline F154-1 & $1.59 \times 10^{-4}$ & $9.62 \times 10^{-4}$ & $2.42 \times 10^{6}$ \\
F154-2 & $1.59 \times 10^{-4}$ & $1.92 \times 10^{-3}$ & $2.75 \times 10^{6}$ \\
F154-3 & $1.59 \times 10^{-4}$ & $2.89 \times 10^{-3}$ & $3.01 \times 10^{6}$ \\
F154-4 & $1.59 \times 10^{-4}$ & $3.85 \times 10^{-3}$ & $3.40 \times 10^{6}$ \\
F154-5 & $1.59 \times 10^{-4}$ & $4.81 \times 10^{-3}$ & $3.55 \times 10^{6}$ \\
\hline
\end{tabular}

${ }^{a} \mathrm{OAc}=$ acetate.

$k_{2}=3.02 \times 10^{8} \mathrm{M}^{-1} \mathrm{~s}^{-1}$

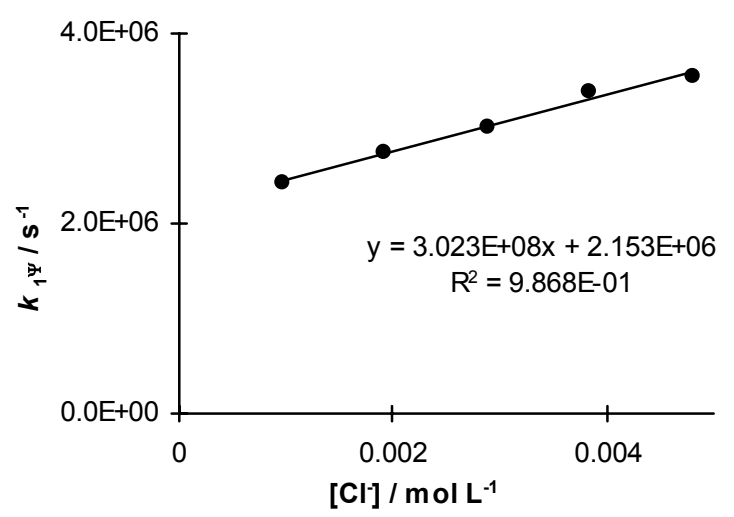


Table S7. (ani) ${ }_{2} \mathrm{CH}^{+}$from (ani) $)_{2} \mathrm{CHOAc}+\mathrm{Cl}^{-}$in $50 \mathrm{E} 50 \mathrm{AN}$ at $20^{\circ} \mathrm{C}$ (Laser flash, detection at $\left.500 \mathrm{~nm}\right){ }^{a}$

\begin{tabular}{llll}
\hline No. & {$[\text { Precursor }]_{0} / \mathrm{M}$} & {$\left[\mathrm{Cl}^{-}\right] / \mathrm{M}$} & $k_{\mathrm{obs}} / \mathrm{s}^{-1}$ \\
\hline F153-1 & $1.59 \times 10^{-4}$ & $1.60 \times 10^{-4}$ & $1.46 \times 10^{6}$ \\
F153-2 & $1.59 \times 10^{-4}$ & $3.21 \times 10^{-4}$ & $1.65 \times 10^{6}$ \\
F153-3 & $1.59 \times 10^{-4}$ & $4.81 \times 10^{-4}$ & $1.98 \times 10^{6}$ \\
F153-4 & $1.59 \times 10^{-4}$ & $6.41 \times 10^{-4}$ & $2.22 \times 10^{6}$ \\
F153-5 & $1.59 \times 10^{-4}$ & $8.02 \times 10^{-4}$ & $2.55 \times 10^{6}$ \\
\hline
\end{tabular}

${ }^{a} \mathrm{OAc}=$ acetate.

$k_{2}=1.71 \times 10^{9} \mathrm{M}^{-1} \mathrm{~s}^{-1}$

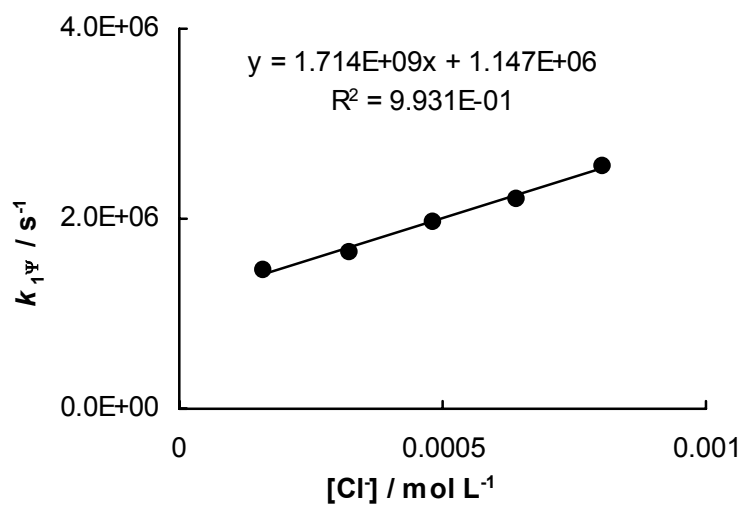


Table S8. (ani) $)_{2} \mathrm{CH}^{+}$from (ani) ${ }_{2} \mathrm{CHOAr}+\mathrm{Cl}^{-}$in $80 \mathrm{~W} 20 \mathrm{AN}$ at $20^{\circ} \mathrm{C}$ (Laser flash, detection at $500 \mathrm{~nm}$ ). ${ }^{a}$

\begin{tabular}{llll}
\hline No. & $\begin{array}{l}{[\text { Precursor }]_{0},} \\
\mathrm{~mol} \mathrm{~L}^{-1}\end{array}$ & $\begin{array}{l}{\left[\mathrm{Cl}^{-}\right]} \\
\mathrm{mol} \mathrm{L}^{-1}\end{array}$ & $\begin{array}{l}r_{1}+r_{2} \\
\mathrm{~s}^{-1}\end{array}$ \\
\hline F174-1 & $1.59 \times 10^{-4}$ & $8.09 \times 10^{-3}$ & $1.57 \times 10^{5}$ \\
F174-2 & $1.59 \times 10^{-4}$ & $1.01 \times 10^{-2}$ & $1.62 \times 10^{5}$ \\
F174-3 & $1.59 \times 10^{-4}$ & $2.43 \times 10^{-2}$ & $2.25 \times 10^{5}$ \\
F174-4 & $1.59 \times 10^{-4}$ & $3.24 \times 10^{-2}$ & $2.41 \times 10^{5}$ \\
\hline
\end{tabular}

${ }^{a} \mathrm{OAr}=4$-cyanophenoxide

$k_{2}=3.23 \times 10^{6} \mathrm{M}^{-1} \mathrm{~s}^{-1}$

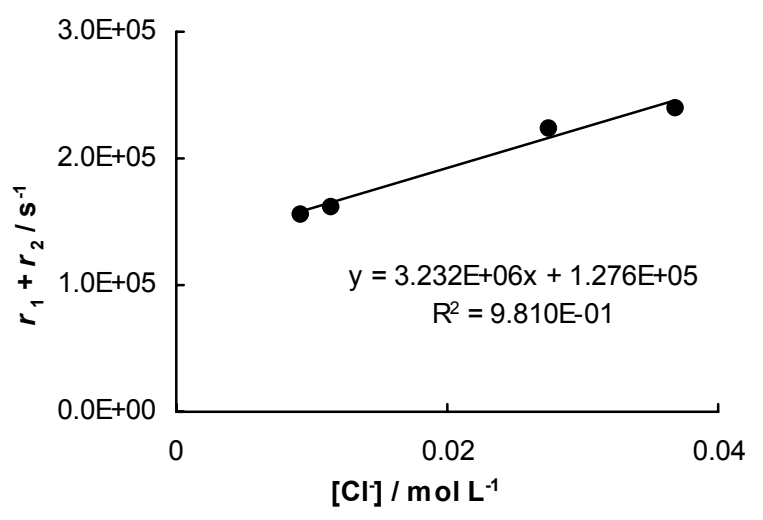


Table S9. (ani) ${ }_{2} \mathrm{CH}^{+}$from (ani) ${ }_{2} \mathrm{CHOAc}+\mathrm{Cl}^{-}$in $50 \mathrm{~W} 50 \mathrm{AN}$ at $20^{\circ} \mathrm{C}$ (Laser flash, detection at $\left.500 \mathrm{~nm}\right){ }^{a}$

\begin{tabular}{llll}
\hline No. & {$[\text { Precursor }]_{0} / \mathrm{M}$} & {$\left[\mathrm{Cl}^{-}\right] / \mathrm{M}$} & $k_{\mathrm{obs}} / \mathrm{s}^{-1}$ \\
\hline F155-1 & $1.59 \times 10^{-4}$ & $9.62 \times 10^{-4}$ & $1.07 \times 10^{5}$ \\
F155-2 & $1.59 \times 10^{-4}$ & $1.92 \times 10^{-3}$ & $1.24 \times 10^{5}$ \\
F155-3 & $1.59 \times 10^{-4}$ & $2.89 \times 10^{-3}$ & $1.36 \times 10^{5}$ \\
F155-4 & $1.59 \times 10^{-4}$ & $3.85 \times 10^{-3}$ & $1.50 \times 10^{5}$ \\
F155-5 & $1.59 \times 10^{-4}$ & $4.81 \times 10^{-3}$ & $1.68 \times 10^{5}$ \\
F155-6 & $1.59 \times 10^{-4}$ & $5.57 \times 10^{-3}$ & $1.80 \times 10^{5}$ \\
F155-7 & $1.59 \times 10^{-4}$ & $6.73 \times 10^{-3}$ & $1.97 \times 10^{5}$ \\
F155-8 & $1.59 \times 10^{-4}$ & $7.69 \times 10^{-3}$ & $2.04 \times 10^{5}$ \\
F155-9 & $1.59 \times 10^{-4}$ & $8.66 \times 10^{-3}$ & $2.26 \times 10^{5}$ \\
F155-10 & $1.59 \times 10^{-4}$ & $9.62 \times 10^{-3}$ & $2.25 \times 10^{5}$
\end{tabular}

${ }^{a} \mathrm{OAc}=$ acetate.

$k_{2}=1.51 \times 10^{7} \mathrm{M}^{-1} \mathrm{~s}^{-1}$

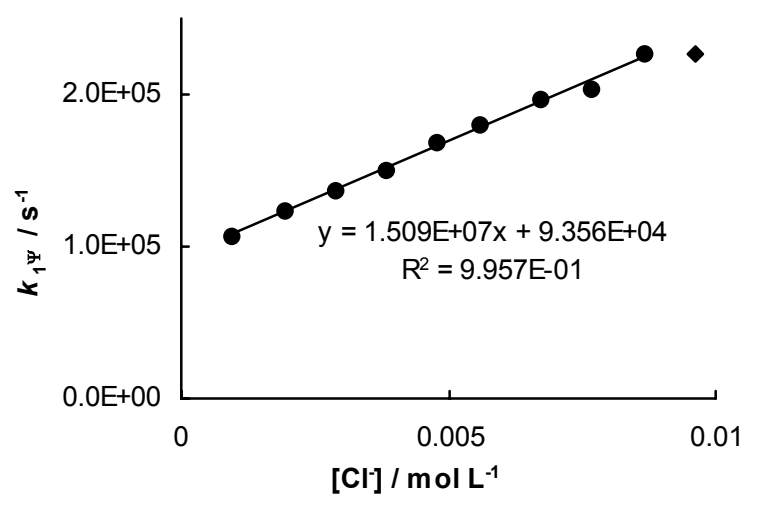


Table S10. (ani)(tol) $\mathrm{CH}^{+}$from (ani)(tol)CHOAr $+\mathrm{Cl}^{-}$in $80 \mathrm{E} 20 \mathrm{~W}$ at $20^{\circ} \mathrm{C}$ (Laser flash, detection at 480 nm). ${ }^{a}$

\begin{tabular}{llll}
\hline No. & {$[\text { Precursor }]_{0} / \mathrm{M}$} & {$\left[\mathrm{Cl}^{-}\right] / \mathrm{M}$} & $k_{\mathrm{obs}} / \mathrm{s}^{-1}$ \\
\hline F118-1 & $2.00 \times 10^{-4}$ & $2.14 \times 10^{-3}$ & $8.94 \times 10^{6}$ \\
F118-2 & $2.00 \times 10^{-4}$ & $4.28 \times 10^{-3}$ & $1.03 \times 10^{7}$ \\
F118-3 & $2.00 \times 10^{-4}$ & $6.42 \times 10^{-3}$ & $1.09 \times 10^{7}$ \\
F118-4 & $2.00 \times 10^{-4}$ & $8.55 \times 10^{-3}$ & $1.21 \times 10^{7}$ \\
F118-5 & $2.00 \times 10^{-4}$ & $1.07 \times 10^{-2}$ & $1.34 \times 10^{7}$ \\
\hline
\end{tabular}

${ }^{a} \mathrm{OAr}=4$-cyanophenoxide

$k_{2}=5.01 \times 10^{8} \mathrm{M}^{-1} \mathrm{~s}^{-1}$

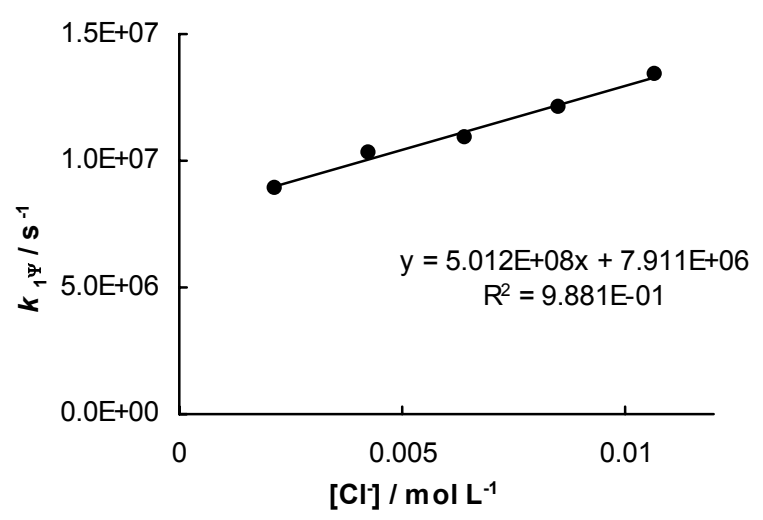


Table S11. (ani)(tol) $\mathrm{CH}^{+}$from (ani)(tol)CHOAc $+\mathrm{Cl}^{-}$in $50 \mathrm{~W} 50 \mathrm{AN}$ at $20^{\circ} \mathrm{C}$ (Laser flash, detection at $480 \mathrm{~nm}){ }^{a}$

\begin{tabular}{llll}
\hline No. & {$[\text { Precursor }]_{0} / \mathrm{M}$} & {$\left[\mathrm{Cl}^{-}\right] / \mathrm{M}$} & $k_{\mathrm{obs}} / \mathrm{s}^{-1}$ \\
\hline F169-1 & $1.50 \times 10^{-4}$ & $9.30 \times 10^{-4}$ & $9.31 \times 10^{5}$ \\
F169-2 & $1.50 \times 10^{-4}$ & $1.86 \times 10^{-3}$ & $1.07 \times 10^{6}$ \\
F169-3 & $1.50 \times 10^{-4}$ & $2.79 \times 10^{-3}$ & $1.18 \times 10^{6}$ \\
F169-4 & $1.50 \times 10^{-4}$ & $3.72 \times 10^{-3}$ & $1.25 \times 10^{6}$ \\
F169-5 & $1.50 \times 10^{-4}$ & $4.65 \times 10^{-3}$ & $1.31 \times 10^{6}$ \\
F169-6 & $1.50 \times 10^{-4}$ & $7.91 \times 10^{-3}$ & $1.54 \times 10^{6}$ \\
F169-7 & $1.50 \times 10^{-4}$ & $1.02 \times 10^{-2}$ & $1.93 \times 10^{6}$ \\
F169-8 & $1.50 \times 10^{-4}$ & $1.49 \times 10^{-2}$ & $2.38 \times 10^{6}$
\end{tabular}

${ }^{a} \mathrm{OAc}=4$-cyanophenoxide

$k_{2}=1.01 \times 10^{8} \mathrm{M}^{-1} \mathrm{~s}^{-1}$

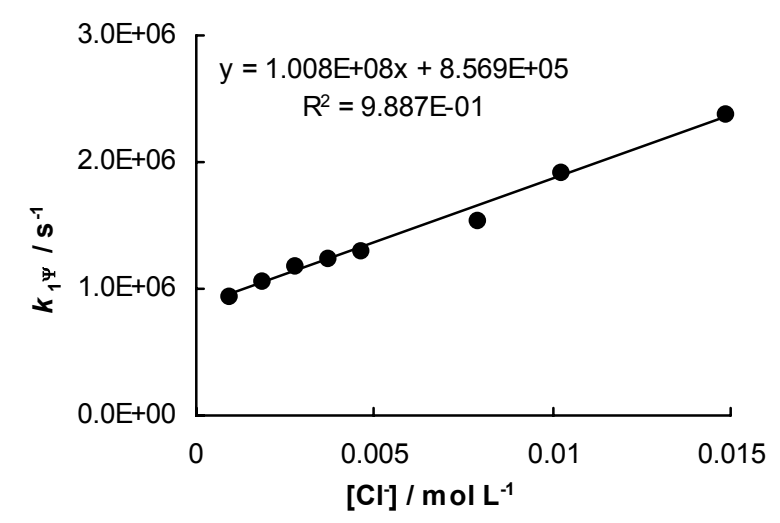


Table S12. (ani)(tol) $\mathrm{CH}^{+}$from (ani)(tol)CHOAc $+\mathrm{Cl}^{-}$in $50 \mathrm{E} 50 \mathrm{~W}$ at $20^{\circ} \mathrm{C}$ (Laser flash, detection at 480 $\mathrm{nm}){ }^{a}$

\begin{tabular}{llll}
\hline No. & {$[\text { Precursor }]_{0} / \mathrm{M}$} & {$\left[\mathrm{Cl}^{-}\right] / \mathrm{M}$} & $k_{\mathrm{obs}} / \mathrm{s}^{-1}$ \\
\hline F163-1 & $1.51 \times 10^{-4}$ & $3.39 \times 10^{-3}$ & $3.14 \times 10^{6}$ \\
F163-2 & $1.51 \times 10^{-4}$ & $6.78 \times 10^{-3}$ & $3.51 \times 10^{6}$ \\
F163-3 & $1.51 \times 10^{-4}$ & $1.02 \times 10^{-2}$ & $3.97 \times 10^{6}$ \\
F163-4 & $1.51 \times 10^{-4}$ & $1.36 \times 10^{-2}$ & $4.49 \times 10^{6}$ \\
F163-5 & $1.51 \times 10^{-4}$ & $1.70 \times 10^{-2}$ & $4.80 \times 10^{6}$ \\
\hline
\end{tabular}

${ }^{a} \mathrm{OAr}=$ acetate

$k_{2}=1.26 \times 10^{8} \mathrm{M}^{-1} \mathrm{~s}^{-1}$

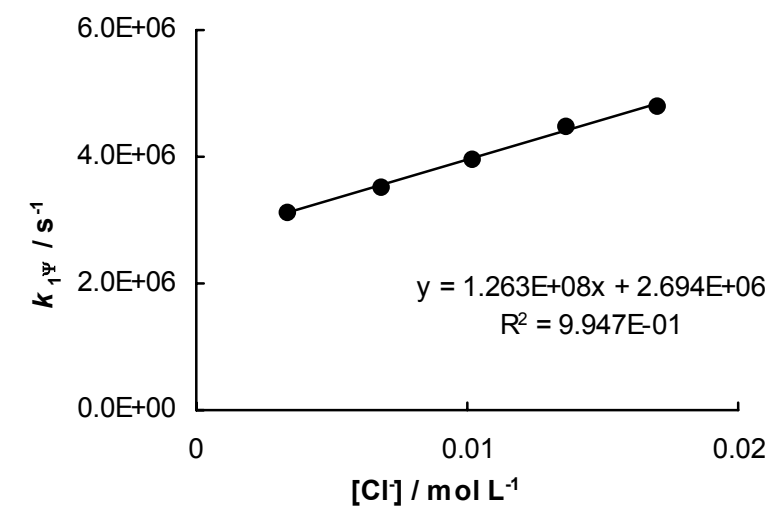


Table S13. (ani) $\mathrm{PhCH}^{+}$from (ani) $\mathrm{PhCHOAr}+\mathrm{Cl}^{-}$in $50 \mathrm{E} 50 \mathrm{~W}$ at $20^{\circ} \mathrm{C}$ (Laser flash, detection at 460 $\mathrm{nm}){ }^{a}$

\begin{tabular}{llll}
\hline No. & {$[\text { Precursor }]_{0} / \mathrm{M}$} & {$\left[\mathrm{Cl}^{-}\right] / \mathrm{M}$} & $k_{\mathrm{obs}} / \mathrm{s}^{-1}$ \\
\hline F164-1 & $1.36 \times 10^{-4}$ & $6.78 \times 10^{-4}$ & $7.82 \times 10^{6}$ \\
F164-2 & $1.36 \times 10^{-4}$ & $1.36 \times 10^{-3}$ & $7.98 \times 10^{6}$ \\
F164-3 & $1.36 \times 10^{-4}$ & $2.03 \times 10^{-3}$ & $8.77 \times 10^{6}$ \\
F164-4 & $1.36 \times 10^{-4}$ & $2.71 \times 10^{-3}$ & $8.72 \times 10^{6}$ \\
F164-5 & $1.36 \times 10^{-4}$ & $3.39 \times 10^{-3}$ & $9.41 \times 10^{6}$ \\
F164-6 & $1.36 \times 10^{-4}$ & $4.07 \times 10^{-3}$ & $9.91 \times 10^{6}$ \\
\hline
\end{tabular}

${ }^{a} \mathrm{OAr}=4$-cyanophenoxide

$k_{2}=6.19 \times 10^{8} \mathrm{M}^{-1} \mathrm{~s}^{-1}$

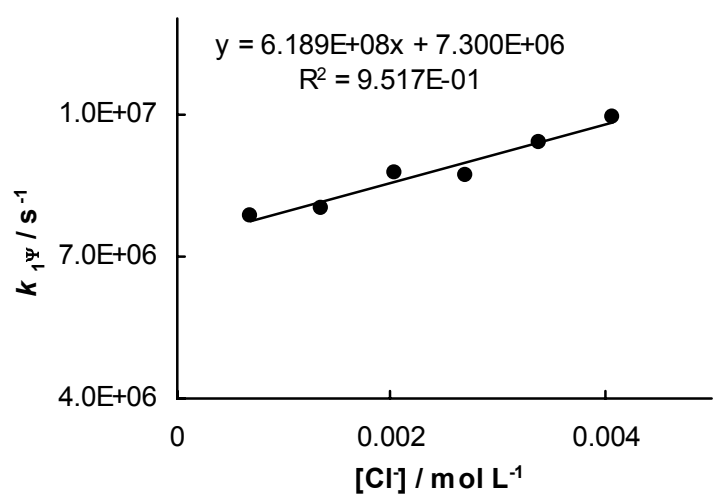


Table S14. (ani) $\mathrm{PhCH}^{+}$from (ani)PhCHOAr $+\mathrm{Cl}^{-}$in 50W50AN at $20^{\circ} \mathrm{C}$ (Laser flash, detection at 460 $\mathrm{nm}){ }^{a}$

\begin{tabular}{llll}
\hline No. & {$[\text { Precursor }]_{0} / \mathrm{M}$} & {$\left[\mathrm{Cl}^{-}\right] / \mathrm{M}$} & $k_{\mathrm{obs}} / \mathrm{s}^{-1}$ \\
\hline F170-1 & $1.36 \times 10^{-4}$ & $4.65 \times 10^{-4}$ & $2.13 \times 10^{6}$ \\
F170-2 & $1.36 \times 10^{-4}$ & $9.30 \times 10^{-4}$ & $2.35 \times 10^{6}$ \\
F170-3 & $1.36 \times 10^{-4}$ & $2.33 \times 10^{-3}$ & $2.75 \times 10^{6}$ \\
F170-4 & $1.36 \times 10^{-4}$ & $3.26 \times 10^{-3}$ & $2.95 \times 10^{6}$ \\
F170-5 & $1.36 \times 10^{-4}$ & $4.65 \times 10^{-3}$ & $3.26 \times 10^{6}$ \\
F170-6 & $1.36 \times 10^{-4}$ & $6.98 \times 10^{-3}$ & $3.42 \times 10^{6}$ \\
F170-7 & $1.36 \times 10^{-4}$ & $9.30 \times 10^{-3}$ & $3.76 \times 10^{6}$ \\
F170-8 & $1.36 \times 10^{-4}$ & $1.16 \times 10^{-2}$ & $4.26 \times 10^{6}$ \\
F170-9 & $1.36 \times 10^{-4}$ & $1.40 \times 10^{-2}$ & $4.44 \times 10^{6}$ \\
\hline
\end{tabular}

${ }^{a} \mathrm{OAr}=4$-cyanophenoxide

$k_{2}=2.64 \times 10^{8} \mathrm{M}^{-1} \mathrm{~s}^{-1}$

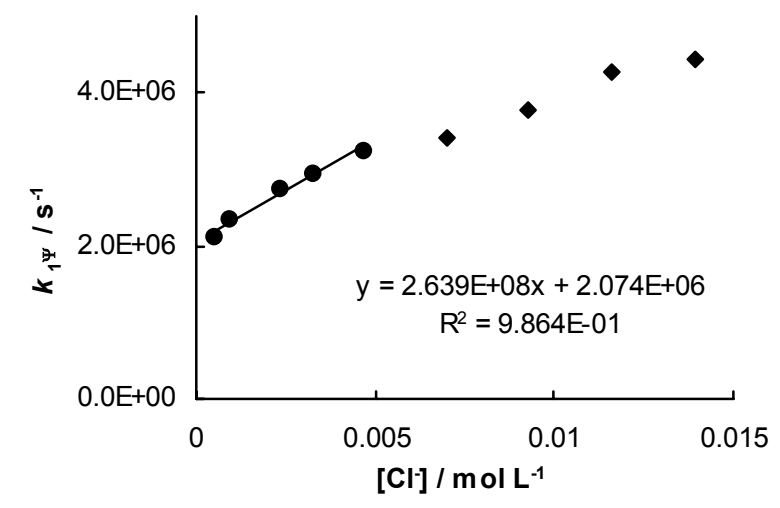


Table S15. (ani) $\mathrm{PhCH}^{+}$from (ani) $\mathrm{PhCHOAr}+\mathrm{Cl}^{-}$in TFE at $20^{\circ} \mathrm{C}$ (Laser flash, detection at $460 \mathrm{~nm}$ ). ${ }^{a}$

\begin{tabular}{llll}
\hline No. & {$[\text { Precursor }]_{0} / \mathrm{M}$} & {$\left[\mathrm{Cl}^{-}\right] / \mathrm{M}$} & $k_{\text {obs }} / \mathrm{s}^{-1}$ \\
\hline F90-1 & $1.78 \times 10^{-4}$ & $2.15 \times 10^{-3}$ & $1.19 \times 10^{5}$ \\
F90-2 & $1.78 \times 10^{-4}$ & $3.94 \times 10^{-3}$ & $1.71 \times 10^{5}$ \\
F90-3 & $1.78 \times 10^{-4}$ & $6.09 \times 10^{-3}$ & $2.33 \times 10^{5}$ \\
F90-4 & $1.78 \times 10^{-4}$ & $7.88 \times 10^{-3}$ & $2.72 \times 10^{5}$ \\
F90-5 & $1.78 \times 10^{-4}$ & $1.00 \times 10^{-2}$ & $3.24 \times 10^{5}$ \\
\hline
\end{tabular}

${ }^{a} \mathrm{OAr}=4$-cyanophenoxide

$k_{2}=2.60 \times 10^{7} \mathrm{M}^{-1} \mathrm{~s}^{-1}$

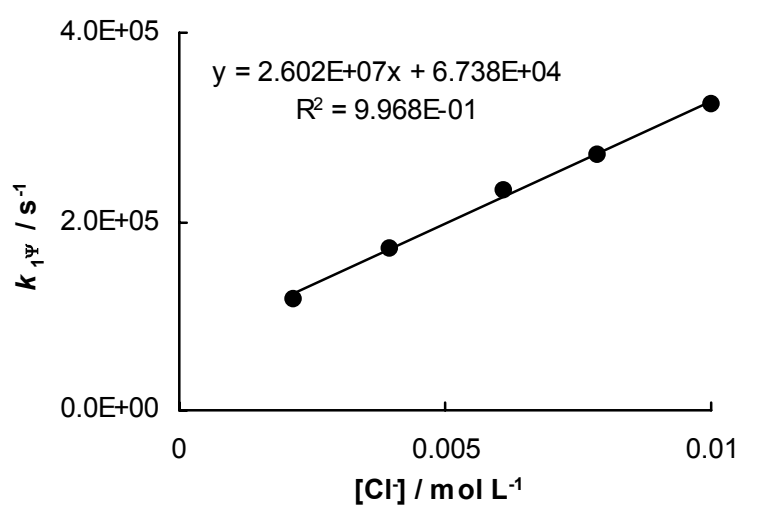


Table S16. (tol) $)_{2} \mathrm{CH}^{+}$from (tol $)_{2} \mathrm{CHOAr}+\mathrm{Cl}^{-}$in TFE at $20^{\circ} \mathrm{C}$ (Laser flash, detection at $\left.460 \mathrm{~nm}\right){ }^{a}$

\begin{tabular}{llll}
\hline No. & {$[\text { Precursor }]_{0} / \mathrm{M}$} & {$\left[\mathrm{Cl}^{-}\right] / \mathrm{M}$} & $k_{\mathrm{obs}} / \mathrm{s}^{-1}$ \\
\hline F84-1 & $9.44 \times 10^{-5}$ & $1.31 \times 10^{-3}$ & $7.40 \times 10^{5}$ \\
F84-2 & $9.44 \times 10^{-5}$ & $2.61 \times 10^{-3}$ & $1.06 \times 10^{6}$ \\
F84-3 & $9.44 \times 10^{-5}$ & $3.92 \times 10^{-3}$ & $1.41 \times 10^{6}$ \\
F84-4 & $9.44 \times 10^{-5}$ & $5.23 \times 10^{-3}$ & $1.73 \times 10^{6}$ \\
F84-5 & $9.44 \times 10^{-5}$ & $6.54 \times 10^{-3}$ & $2.06 \times 10^{6}$ \\
\hline
\end{tabular}

${ }^{a} \mathrm{OAr}=4$-cyanophenoxide

$k_{2}=2.53 \times 10^{8} \mathrm{M}^{-1} \mathrm{~s}^{-1}$

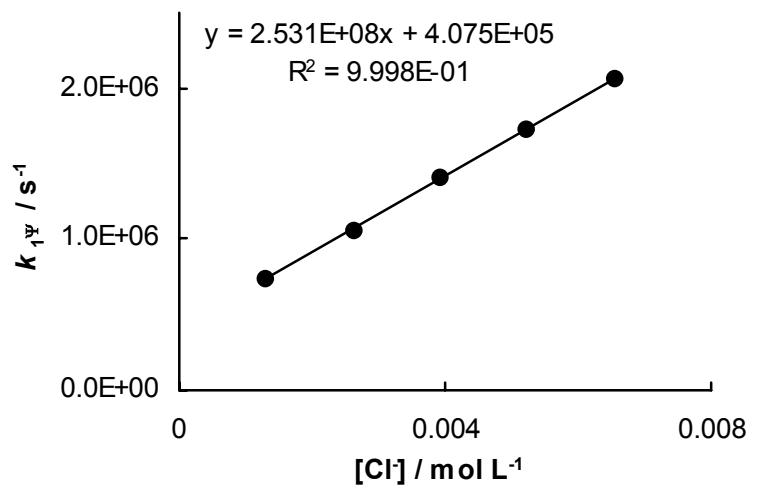


Table S17. (tol) $\mathrm{PhCH}^{+}$from (tol)PhCHOAr $+\mathrm{Cl}^{-}$in TFE at $20^{\circ} \mathrm{C}$ (Laser flash, detection at $\left.450 \mathrm{~nm}\right){ }^{a}$

\begin{tabular}{llll}
\hline No. & {$[\text { Precursor }]_{0} / \mathrm{M}$} & {$\left[\mathrm{Cl}^{-}\right] / \mathrm{M}$} & $k_{\mathrm{obs}} / \mathrm{s}^{-1}$ \\
\hline F92-1 & $1.74 \times 10^{-4}$ & $7.16 \times 10^{-4}$ & $1.59 \times 10^{6}$ \\
F92-2 & $1.74 \times 10^{-4}$ & $1.59 \times 10^{-3}$ & $2.83 \times 10^{6}$ \\
F92-3 & $1.74 \times 10^{-4}$ & $1.07 \times 10^{-3}$ & $2.08 \times 10^{6}$ \\
F92-4 & $1.74 \times 10^{-4}$ & $2.51 \times 10^{-3}$ & $3.51 \times 10^{6}$ \\
F92-5 & $1.74 \times 10^{-4}$ & $3.04 \times 10^{-3}$ & $3.82 \times 10^{6}$ \\
F92-6 & $1.74 \times 10^{-4}$ & $3.58 \times 10^{-3}$ & $4.80 \times 10^{6}$ \\
F92-7 & $1.74 \times 10^{-4}$ & $4.30 \times 10^{-3}$ & $5.64 \times 10^{6}$ \\
\hline
\end{tabular}

${ }^{a} \mathrm{OAr}=4$-cyanophenoxide

$k_{2}=1.07 \times 10^{9} \mathrm{M}^{-1} \mathrm{~s}^{-1}$

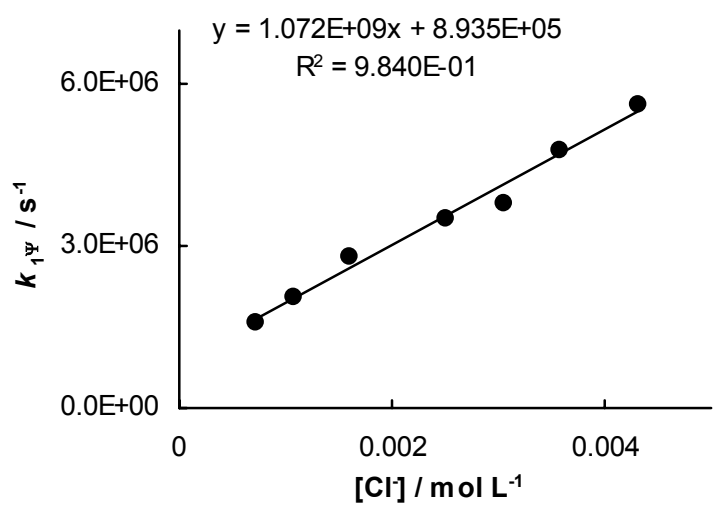


Table S18. (tol) $\mathrm{PhCH}^{+}$from (tol)PhCHOAr $+\mathrm{Cl}^{-}$in $\mathrm{HFIP}$ at $20^{\circ} \mathrm{C}$ (Laser flash, detection at $455 \mathrm{~nm}$ ). ${ }^{a}$

\begin{tabular}{llll}
\hline No. & {$[\text { Precursor }]_{0} / \mathrm{M}$} & {$\left[\mathrm{Cl}^{-}\right] / \mathrm{M}$} & $k_{\mathrm{obs}} / \mathrm{s}^{-1}$ \\
\hline F139-1 & $2.88 \times 10^{-4}$ & $2.23 \times 10^{-3}$ & $1.52 \times 10^{5}$ \\
F139-2 & $2.88 \times 10^{-4}$ & $4.46 \times 10^{-3}$ & $2.32 \times 10^{5}$ \\
F139-3 & $2.88 \times 10^{-4}$ & $8.92 \times 10^{-3}$ & $3.85 \times 10^{5}$ \\
F139-4 & $2.88 \times 10^{-4}$ & $1.12 \times 10^{-2}$ & $4.30 \times 10^{5}$ \\
F139-5 & $2.88 \times 10^{-4}$ & $1.20 \times 10^{-2}$ & $4.16 \times 10^{5}$ \\
F139-6 & $2.88 \times 10^{-4}$ & $1.34 \times 10^{-2}$ & $4.30 \times 10^{5}$ \\
F139-7 & $2.88 \times 10^{-4}$ & $2.68 \times 10^{-2}$ & $6.53 \times 10^{5}$ \\
\hline
\end{tabular}

${ }^{a} \mathrm{OAr}=4$-cyanophenoxide

$k_{2}=3.47 \times 10^{7} \mathrm{M}^{-1} \mathrm{~s}^{-1}$

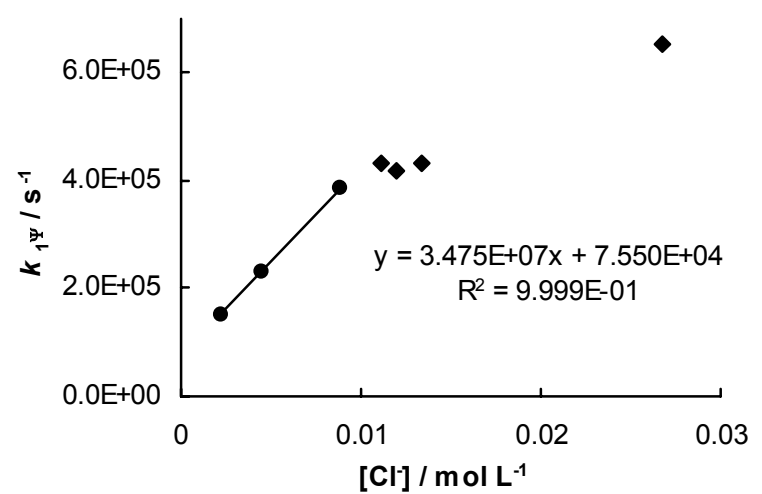


Table S19. $\mathrm{Ph}_{2} \mathrm{CH}^{+}$from $\mathrm{Ph}_{2} \mathrm{CHOAr}+\mathrm{Cl}^{-}$in $\mathrm{HFIP}$ at $20^{\circ} \mathrm{C}$ (Laser flash, detection at $\left.440 \mathrm{~nm}\right){ }^{a}$

\begin{tabular}{llll}
\hline No. & {$[\text { Precursor }]_{0} / \mathrm{M}$} & {$\left[\mathrm{Cl}^{-}\right] / \mathrm{M}$} & $k_{\mathrm{obs}} / \mathrm{s}^{-1}$ \\
\hline F138-1 & $2.23 \times 10^{-4}$ & $2.47 \times 10^{-4}$ & $2.67 \times 10^{5}$ \\
F138-2 & $2.23 \times 10^{-4}$ & $4.94 \times 10^{-4}$ & $4.27 \times 10^{5}$ \\
F138-3 & $2.23 \times 10^{-4}$ & $7.42 \times 10^{-4}$ & $5.67 \times 10^{5}$ \\
F138-4 & $2.23 \times 10^{-4}$ & $9.89 \times 10^{-4}$ & $7.08 \times 10^{5}$ \\
F138-5 & $2.23 \times 10^{-4}$ & $1.24 \times 10^{-3}$ & $8.85 \times 10^{5}$ \\
F138-6 & $2.23 \times 10^{-4}$ & $2.47 \times 10^{-3}$ & $1.36 \times 10^{6}$ \\
F138-7 & $2.23 \times 10^{-4}$ & $3.71 \times 10^{-3}$ & $1.88 \times 10^{6}$ \\
F138-8 & $2.23 \times 10^{-4}$ & $4.94 \times 10^{-3}$ & $2.18 \times 10^{6}$ \\
F138-9 & $2.23 \times 10^{-4}$ & $6.18 \times 10^{-3}$ & $2.26 \times 10^{6}$ \\
\hline
\end{tabular}

${ }^{a} \mathrm{OAr}=4$-cyanophenoxide

$k_{2}=6.12 \times 10^{8} \mathrm{M}^{-1} \mathrm{~s}^{-1}$

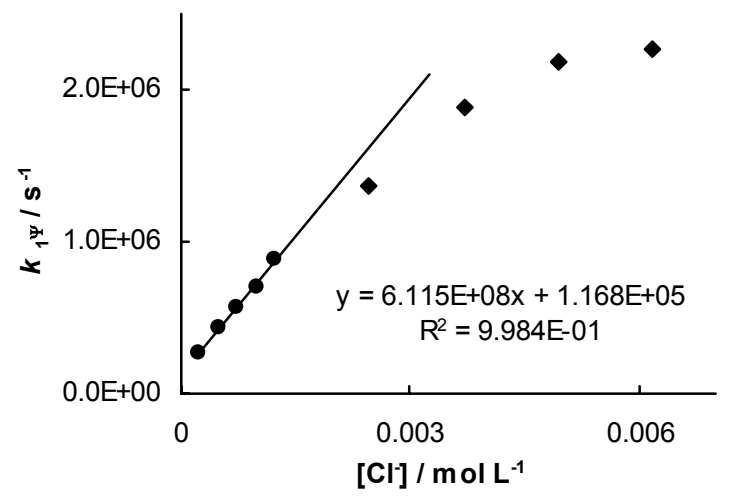


Table S20. Rate constants for the reaction of (fur) $)_{2} \mathrm{CHCl}$ with $n \mathrm{Bu}_{4} \mathrm{~N}^{+} \mathrm{Cl}^{-}$in $\mathrm{AN}$ by Laser flash $(\lambda=520$ $\left.\mathrm{nm}, 20^{\circ} \mathrm{C}\right)$.

\begin{tabular}{ccll}
\hline Nr. & $\begin{array}{c}{[\mathrm{E}]_{0} /} \\
\mathrm{mol} \mathrm{L}\end{array}$ & $\begin{array}{c}{[\mathrm{Nu}]_{0} /} \\
\mathrm{mol} \mathrm{L}\end{array}$ & $\begin{array}{c}k_{\psi} / \\
\mathrm{s}^{-1}\end{array}$ \\
\hline 14030201 & $3.59 \times 10^{-5}$ & 0.00 & $1.16 \times 10^{5}$ \\
14030202 & $3.59 \times 10^{-5}$ & $2.72 \times 10^{-4}$ & $2.89 \times 10^{6}$ \\
13030207 & $7.17 \times 10^{-5}$ & $2.76 \times 10^{-4}$ & $3.60 \times 10^{6}$ \\
19030207 & $7.17 \times 10^{-5}$ & $3.03 \times 10^{-4}$ & $3.31 \times 10^{6}$ \\
14030203 & $3.59 \times 10^{-5}$ & $5.43 \times 10^{-4}$ & $6.78 \times 10^{6}$ \\
13030204 & $7.17 \times 10^{-5}$ & $5.51 \times 10^{-4}$ & $5.78 \times 10^{6}$ \\
19030208 & $7.17 \times 10^{-5}$ & $5.68 \times 10^{-4}$ & $5.59 \times 10^{6}$ \\
19030209 & $7.17 \times 10^{-5}$ & $7.57 \times 10^{-4}$ & $7.46 \times 10^{6}$ \\
14030204 & $3.59 \times 10^{-5}$ & $8.15 \times 10^{-4}$ & $8.66 \times 10^{6}$ \\
14030205 & $3.59 \times 10^{-5}$ & $1.09 \times 10^{-3}$ & $1.09 \times 10^{7}$ \\
13030205 & $7.17 \times 10^{-5}$ & $1.10 \times 10^{-3}$ & $1.11 \times 10^{7}$ \\
19030210 & $7.17 \times 10^{-5}$ & $1.14 \times 10^{-3}$ & $1.05 \times 10^{7}$ \\
13030206 & $7.17 \times 10^{-5}$ & $1.65 \times 10^{-3}$ & $1.62 \times 10^{7}$ \\
\hline
\end{tabular}

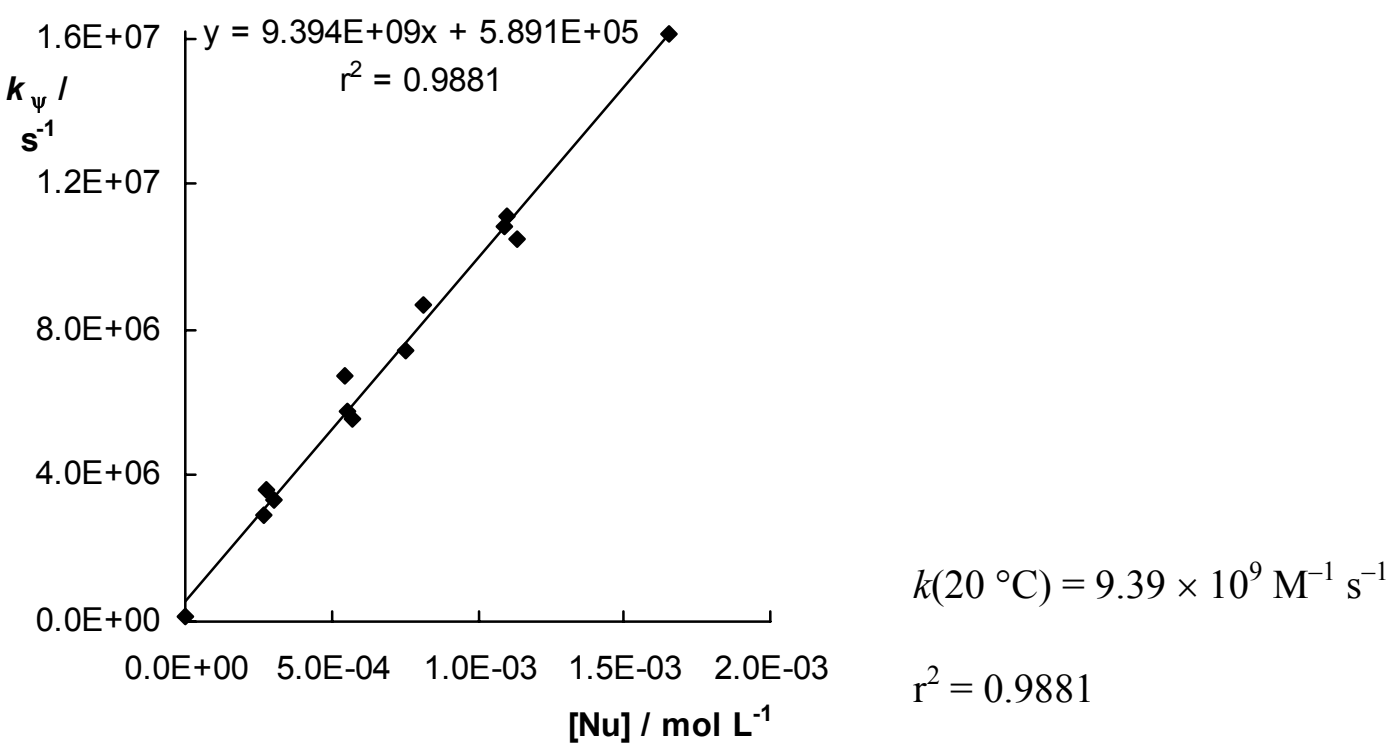


Table 21. Rate constants for the reaction of $(\mathrm{pfa})_{2} \mathrm{CH}^{+} \mathrm{BF}_{4}^{-}$with $n \mathrm{Bu}_{4} \mathrm{~N}^{+} \mathrm{Cl}$ in $\mathrm{AN}$ by Laser flash $(\lambda=$ $592 \mathrm{~nm}, 20^{\circ} \mathrm{C}$ ).

\begin{tabular}{cccc}
\hline Nr. & $\begin{array}{c}{[\mathrm{E}]_{0} /} \\
\mathrm{mol} \mathrm{L}^{-1}\end{array}$ & $\begin{array}{c}{[\mathrm{Nu}]_{0} /} \\
\mathrm{mol} \mathrm{L}^{-1}\end{array}$ & $\begin{array}{c}k_{\psi} / \\
\mathrm{s}^{-1}\end{array}$ \\
\hline 01040201 & $7.16 \times 10^{-5}$ & $3.61 \times 10^{-4}$ & $4.58 \times 10^{5}$ \\
01040202 & $7.16 \times 10^{-5}$ & $7.21 \times 10^{-4}$ & $8.61 \times 10^{5}$ \\
01040203 & $7.16 \times 10^{-5}$ & $1.08 \times 10^{-3}$ & $1.26 \times 10^{6}$ \\
01040204 & $7.16 \times 10^{-5}$ & $1.44 \times 10^{-3}$ & $1.52 \times 10^{6}$ \\
01040205 & $4.30 \times 10^{-5}$ & $2.16 \times 10^{-3}$ & $2.25 \times 10^{6}$ \\
01040206 & $4.30 \times 10^{-5}$ & $2.89 \times 10^{-3}$ & $2.94 \times 10^{6}$ \\
\hline
\end{tabular}

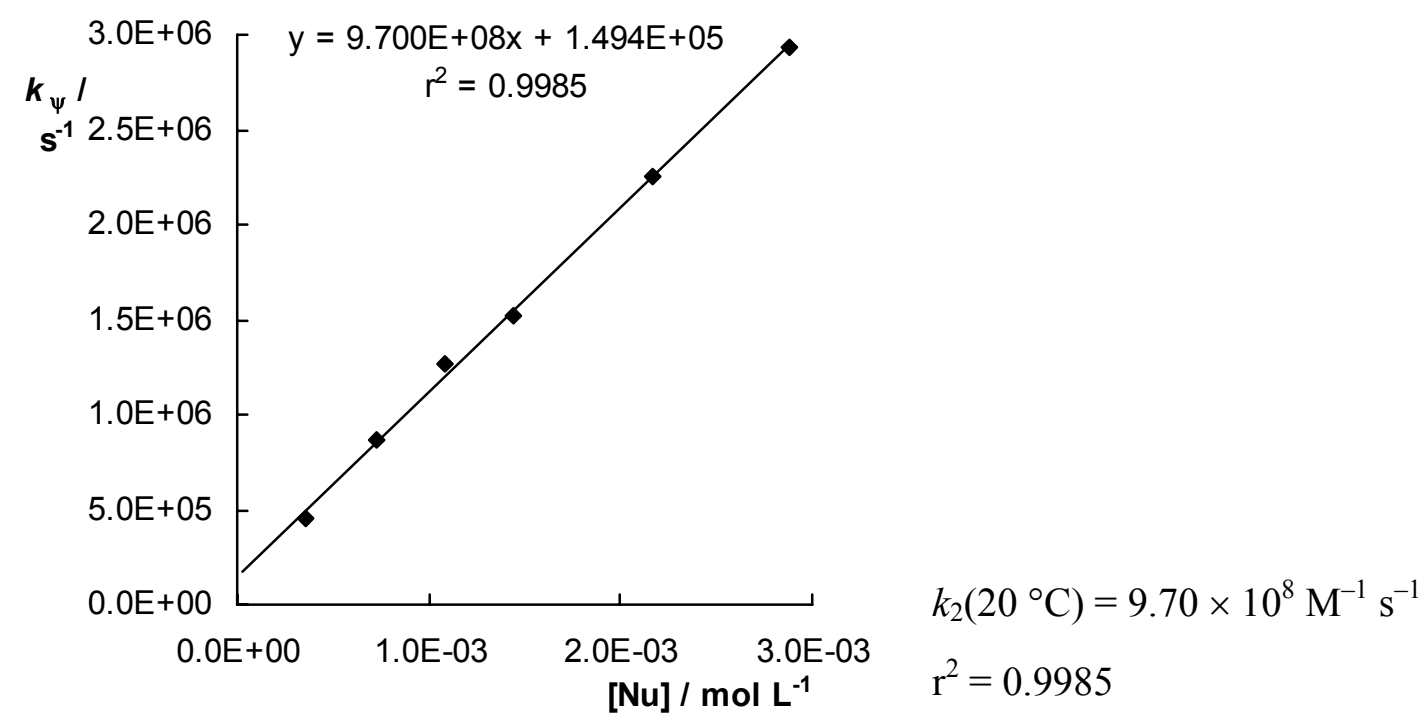


Table S22. Rate constants for the reaction of $(\mathrm{mfa})_{2} \mathrm{CH}^{+} \mathrm{BF}_{4}^{-}$with $n \mathrm{Bu}_{4} \mathrm{~N}^{+} \mathrm{Cl}$ in $\mathrm{AN}$ by Laser flash $(\lambda=$ $\left.586 \mathrm{~nm}, 20^{\circ} \mathrm{C}\right)$.

\begin{tabular}{cccc}
\hline Nr. & $\begin{array}{c}{[\mathrm{E}]_{0} /} \\
\mathrm{mol} \mathrm{L}^{-1}\end{array}$ & $\begin{array}{c}{[\mathrm{Nu}]_{0} /} \\
\mathrm{mol} \mathrm{L}^{-1}\end{array}$ & $\begin{array}{c}k_{\psi} / \\
\mathrm{s}^{-1}\end{array}$ \\
\hline 08040201 & $4.23 \times 10^{-5}$ & $1.14 \times 10^{-3}$ & $3.82 \times 10^{5}$ \\
08040202 & $2.11 \times 10^{-5}$ & $1.14 \times 10^{-3}$ & $3.47 \times 10^{5}$ \\
08040204 & $2.11 \times 10^{-5}$ & $2.28 \times 10^{-3}$ & $5.62 \times 10^{5}$ \\
08040205 & $2.11 \times 10^{-5}$ & $3.42 \times 10^{-3}$ & $7.25 \times 10^{5}$ \\
08040206 & $2.11 \times 10^{-5}$ & $4.57 \times 10^{-3}$ & $9.20 \times 10^{5}$ \\
\hline
\end{tabular}

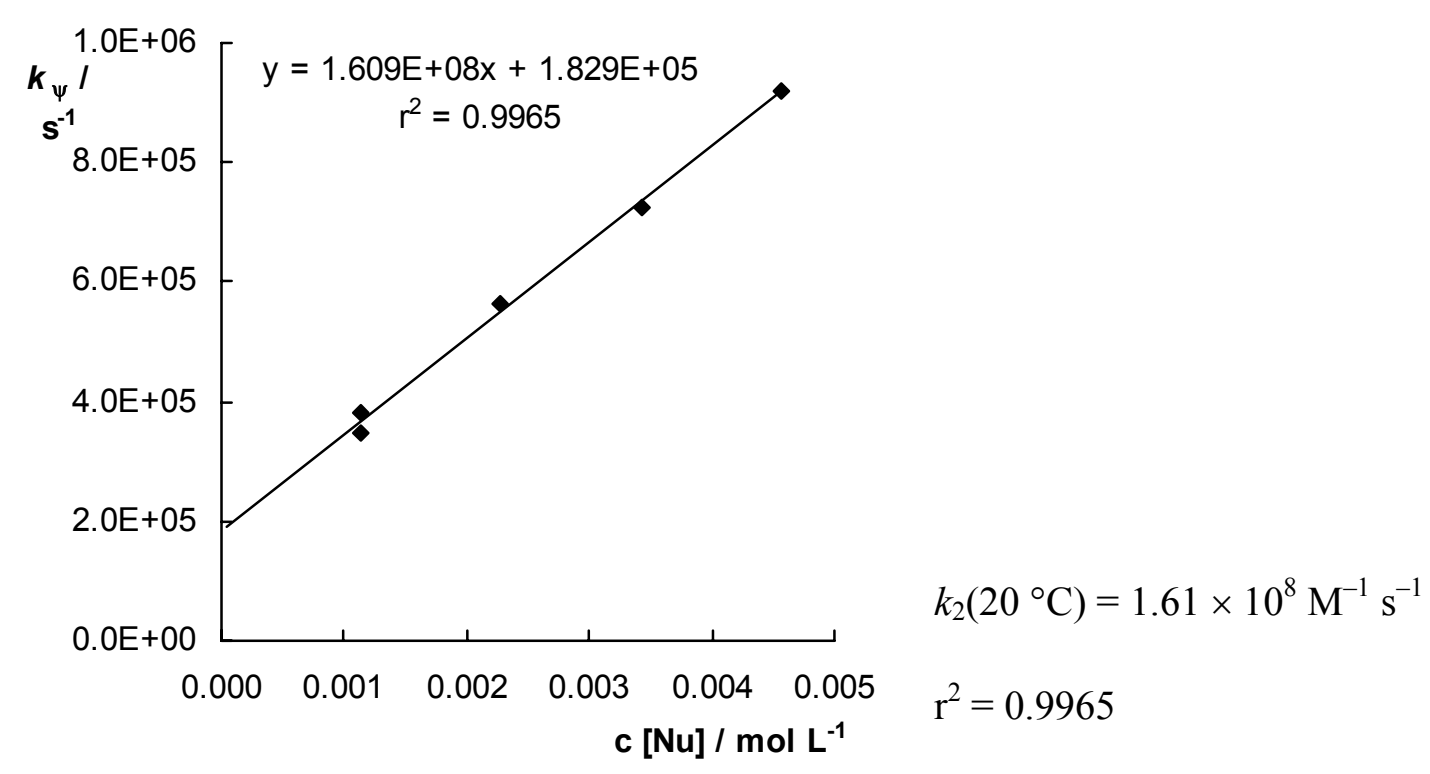


Table S23. Rate constants for the reaction of $(\mathrm{dpa})_{2} \mathrm{CH}^{+} \mathrm{BF}_{4}^{-}$with $n \mathrm{Bu}_{4} \mathrm{~N}^{+} \mathrm{Cl}$ in $\mathrm{AN}$ by Laser flash $(\lambda=$ $\left.586 \mathrm{~nm}, 20^{\circ} \mathrm{C}\right)$.

\begin{tabular}{cccc}
\hline Nr. & $\begin{array}{c}{[\mathrm{E}]_{0} /} \\
\mathrm{mol} \mathrm{L}^{-1}\end{array}$ & $\begin{array}{c}{[\mathrm{Nu}]_{0} /} \\
\mathrm{mol} \mathrm{L}^{-1}\end{array}$ & $\begin{array}{c}k_{\psi} / \\
\mathrm{s}^{-1}\end{array}$ \\
\hline 25030202 & $7.16 \times 10^{-5}$ & $7.94 \times 10^{-3}$ & $2.07 \times 10^{5}$ \\
25030203 & $7.16 \times 10^{-5}$ & $1.19 \times 10^{-2}$ & $3.05 \times 10^{5}$ \\
25030204 & $7.16 \times 10^{-5}$ & $1.98 \times 10^{-2}$ & $4.38 \times 10^{5}$ \\
25030205 & $7.16 \times 10^{-5}$ & $2.98 \times 10^{-2}$ & $5.73 \times 10^{5}$ \\
25030206 & $7.16 \times 10^{-5}$ & $3.97 \times 10^{-2}$ & $7.92 \times 10^{5}$ \\
\hline
\end{tabular}

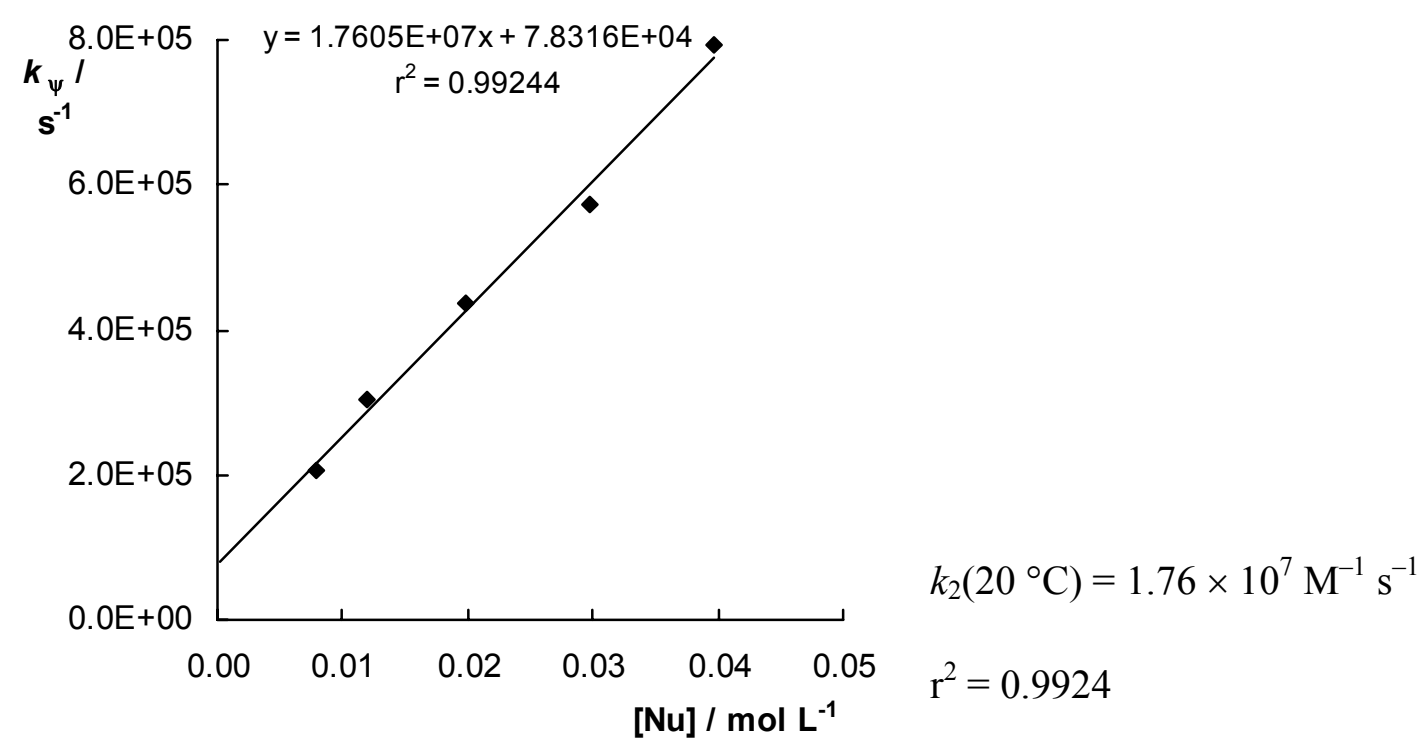




\section{Reactions with bromide $\left(n \mathrm{Bu}_{4} \mathrm{~N}^{+} \mathrm{Br}^{-}\right)$}

Table S24. (ani) $)_{2} \mathrm{CH}^{+}$from (ani) $)_{2} \mathrm{CHOAr}+\mathrm{Br}^{-}$in methanol at $20^{\circ} \mathrm{C}$ (Laser flash, detection at $\left.500 \mathrm{~nm}\right){ }^{a}$

\begin{tabular}{llll}
\hline No. & {$[\text { Precursor }]_{0} / \mathrm{M}$} & {$\left[\mathrm{Br}^{-}\right] / \mathrm{M}$} & $k_{\mathrm{obs}} / \mathrm{s}^{-1}$ \\
\hline F67-1 & $1.99 \times 10^{-4}$ & $1.09 \times 10^{-3}$ & $6.75 \times 10^{6}$ \\
F67-2 & $1.99 \times 10^{-4}$ & $2.03 \times 10^{-3}$ & $8.28 \times 10^{6}$ \\
F67-3 & $1.99 \times 10^{-4}$ & $3.60 \times 10^{-3}$ & $8.88 \times 10^{6}$ \\
F67-4 & $1.99 \times 10^{-4}$ & $5.00 \times 10^{-3}$ & $8.67 \times 10^{6}$ \\
F67-5 & $1.99 \times 10^{-4}$ & $6.57 \times 10^{-3}$ & $1.08 \times 10^{7}$ \\
F67-6 & $1.99 \times 10^{-4}$ & $7.82 \times 10^{-3}$ & $1.14 \times 10^{7}$ \\
F67-7 & $1.99 \times 10^{-4}$ & $1.02 \times 10^{-2}$ & $1.18 \times 10^{7}$ \\
F67-8 & $1.99 \times 10^{-4}$ & $1.33 \times 10^{-2}$ & $1.43 \times 10^{7}$ \\
F67-9 & $1.99 \times 10^{-4}$ & $1.56 \times 10^{-2}$ & $1.55 \times 10^{7}$ \\
F67-10 & $1.99 \times 10^{-4}$ & $1.80 \times 10^{-2}$ & $1.58 \times 10^{7}$ \\
F67-11 & $1.99 \times 10^{-4}$ & $2.03 \times 10^{-2}$ & $1.74 \times 10^{7}$ \\
\hline
\end{tabular}

${ }^{a} \mathrm{OAr}=4$-cyanophenoxide

$k_{2}=5.31 \times 10^{8} \mathrm{M}^{-1} \mathrm{~s}^{-1}$

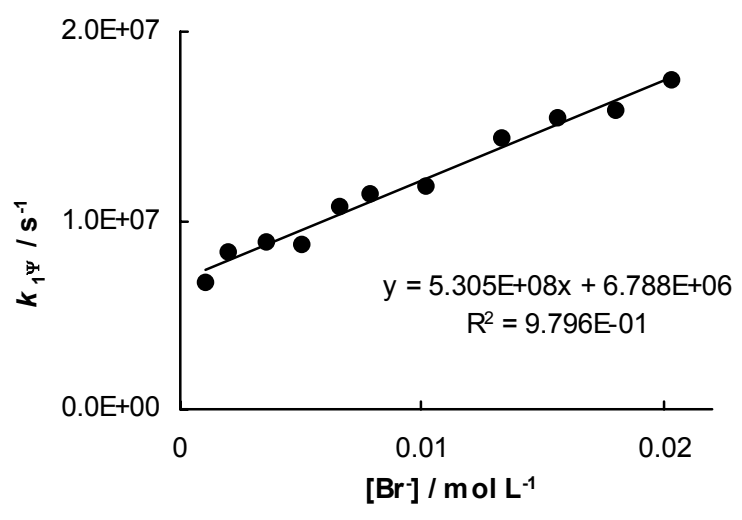


Table S25. (ani) $)_{2} \mathrm{CH}^{+}$from (ani) $)_{2} \mathrm{CHOAr}+\mathrm{Br}^{-}$in ethanol at $20^{\circ} \mathrm{C}$ (Laser flash, detection at $\left.500 \mathrm{~nm}\right){ }^{a}$

\begin{tabular}{llll}
\hline No. & {$[\text { Precursor }]_{0} / \mathrm{M}$} & {$\left[\mathrm{Br}^{-}\right] / \mathrm{M}$} & $k_{\mathrm{obs}} / \mathrm{s}^{-1}$ \\
\hline F71-1 & $1.99 \times 10^{-4}$ & $8.91 \times 10^{-4}$ & $7.94 \times 10^{6}$ \\
F71-2 & $1.99 \times 10^{-4}$ & $1.78 \times 10^{-3}$ & $1.22 \times 10^{7}$ \\
F71-3 & $1.99 \times 10^{-4}$ & $2.67 \times 10^{-3}$ & $1.51 \times 10^{7}$ \\
F71-4 & $1.99 \times 10^{-4}$ & $2.23 \times 10^{-3}$ & $1.33 \times 10^{7}$ \\
F71-5 & $1.99 \times 10^{-4}$ & $3.56 \times 10^{-3}$ & $1.65 \times 10^{7}$ \\
F71-6 & $1.99 \times 10^{-4}$ & $4.45 \times 10^{-3}$ & $1.83 \times 10^{7}$ \\
F71-7 & $1.99 \times 10^{-4}$ & $5.34 \times 10^{-3}$ & $2.23 \times 10^{7}$ \\
F71-8 & $1.99 \times 10^{-4}$ & $6.68 \times 10^{-3}$ & $2.45 \times 10^{7}$ \\
\hline
\end{tabular}

${ }^{a} \mathrm{OAr}=4$-cyanophenoxide

$k_{2}=2.75 \times 10^{9} \mathrm{M}^{-1} \mathrm{~s}^{-1}$

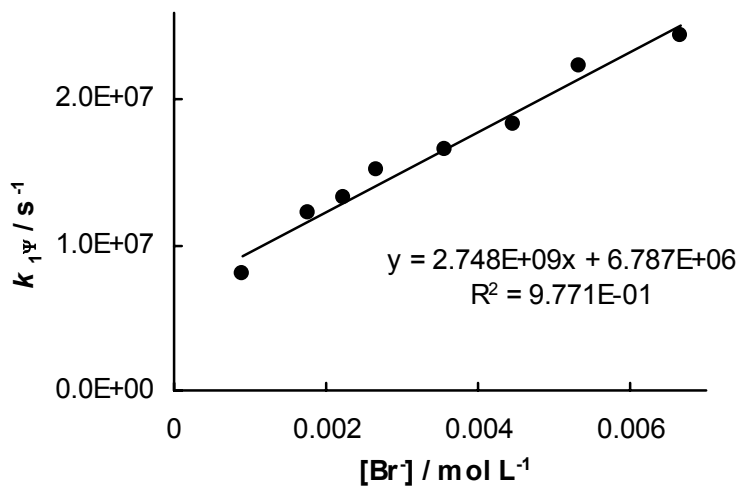


Table S26. (ani) $)_{2} \mathrm{CH}^{+}$from (ani) $)_{2} \mathrm{CHOAr}+\mathrm{Br}^{-}$in $80 \mathrm{E} 20 \mathrm{~W}$ at $20^{\circ} \mathrm{C}$ (Laser flash, detection at $\left.500 \mathrm{~nm}\right){ }^{a}$

\begin{tabular}{llll}
\hline No. & {$[\text { Precursor }]_{0} / \mathrm{M}$} & {$\left[\mathrm{Br}^{-}\right] / \mathrm{M}$} & $k_{\mathrm{obs}} / \mathrm{s}^{-1}$ \\
\hline F112-1 & $1.11 \times 10^{-4}$ & $1.04 \times 10^{-3}$ & $2.21 \times 10^{6}$ \\
F112-2 & $1.11 \times 10^{-4}$ & $2.08 \times 10^{-3}$ & $2.69 \times 10^{6}$ \\
F112-3 & $1.11 \times 10^{-4}$ & $3.12 \times 10^{-3}$ & $3.18 \times 10^{6}$ \\
F112-4 & $1.11 \times 10^{-4}$ & $4.17 \times 10^{-3}$ & $3.48 \times 10^{6}$ \\
F112-5 & $1.11 \times 10^{-4}$ & $5.21 \times 10^{-3}$ & $4.19 \times 10^{6}$ \\
F112-6 & $1.11 \times 10^{-4}$ & $6.25 \times 10^{-3}$ & $4.78 \times 10^{6}$
\end{tabular}

${ }^{a} \mathrm{OAr}=4$-cyanophenoxide

$k_{2}=4.84 \times 10^{8} \mathrm{M}^{-1} \mathrm{~s}^{-1}$

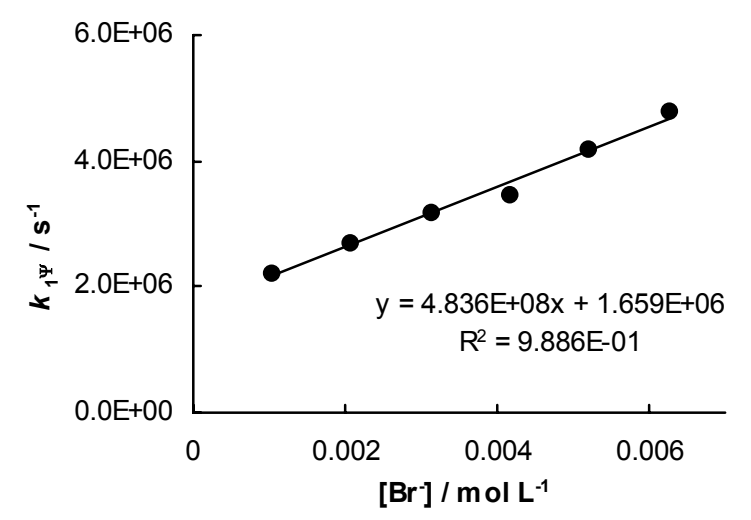


Table S27. (ani) ${ }_{2} \mathrm{CH}^{+}$from (ani) $)_{2} \mathrm{CHOAc}+\mathrm{Br}^{-}$in $50 \mathrm{E} 50 \mathrm{~W}$ at $20^{\circ} \mathrm{C}$ (Laser flash, detection at $\left.500 \mathrm{~nm}\right){ }^{a}$

\begin{tabular}{llll}
\hline No. & $\begin{array}{l}{[\text { Precursor }]_{0},} \\
\mathrm{~mol} \mathrm{~L}^{-1}\end{array}$ & $\begin{array}{l}{\left[\mathrm{Br}^{-}\right]} \\
\mathrm{mol} \mathrm{L}^{-1}\end{array}$ & $\begin{array}{l}r_{1}+r_{2} \\
\mathrm{~s}^{-1}\end{array}$ \\
\hline F165-1 & $1.59 \times 10^{-4}$ & $1.64 \times 10^{-3}$ & $1.08 \times 10^{6}$ \\
F165-2 & $1.59 \times 10^{-4}$ & $3.27 \times 10^{-3}$ & $1.21 \times 10^{6}$ \\
F165-3 & $1.59 \times 10^{-4}$ & $4.91 \times 10^{-3}$ & $1.52 \times 10^{6}$ \\
F165-4 & $1.59 \times 10^{-4}$ & $6.55 \times 10^{-3}$ & $1.52 \times 10^{6}$ \\
F165-5 & $1.59 \times 10^{-4}$ & $8.19 \times 10^{-3}$ & $1.94 \times 10^{6}$ \\
F165-6 & $1.59 \times 10^{-4}$ & $1.15 \times 10^{-2}$ & $2.39 \times 10^{6}$ \\
F165-7 & $1.59 \times 10^{-4}$ & $1.47 \times 10^{-2}$ & $2.79 \times 10^{6}$ \\
F165-8 & $1.59 \times 10^{-4}$ & $1.80 \times 10^{-2}$ & $3.28 \times 10^{6}$ \\
\hline
\end{tabular}

${ }^{a} \mathrm{OAr}=$ acetate

$k_{2}=1.37 \times 10^{8} \mathrm{M}^{-1} \mathrm{~s}^{-1}$

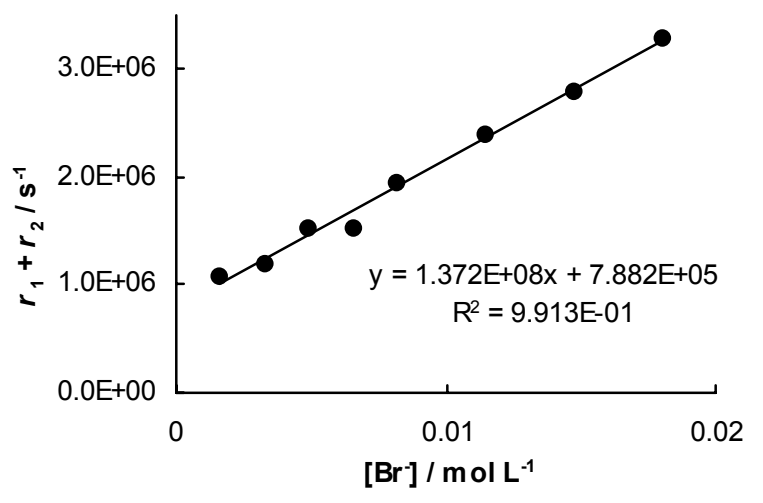


Table S28. (ani) $)_{2} \mathrm{CH}^{+}$from (ani) $)_{2} \mathrm{CHOAc}+\mathrm{Br}^{-}$in $50 \mathrm{M} 50 \mathrm{AN}$ at $20^{\circ} \mathrm{C}$ (Laser flash, detection at 500 $\mathrm{nm}){ }^{a}$

\begin{tabular}{llll}
\hline No. & {$[\text { Precursor }]_{0} / \mathrm{M}$} & {$\left[\mathrm{Br}^{-}\right] / \mathrm{M}$} & $k_{\mathrm{obs}} / \mathrm{s}^{-1}$ \\
\hline F159-1 & $1.59 \times 10^{-4}$ & $2.70 \times 10^{-4}$ & $2.87 \times 10^{6}$ \\
F159-2 & $1.59 \times 10^{-4}$ & $5.40 \times 10^{-4}$ & $3.14 \times 10^{6}$ \\
F159-3 & $1.59 \times 10^{-4}$ & $8.10 \times 10^{-4}$ & $3.69 \times 10^{6}$ \\
F159-4 & $1.59 \times 10^{-4}$ & $1.08 \times 10^{-3}$ & $4.06 \times 10^{6}$ \\
F159-5 & $1.59 \times 10^{-4}$ & $1.35 \times 10^{-3}$ & $4.61 \times 10^{6}$ \\
\hline
\end{tabular}

${ }^{a} \mathrm{OAc}=$ acetate

$k_{2}=1.63 \times 10^{9} \mathrm{M}^{-1} \mathrm{~s}^{-1}$

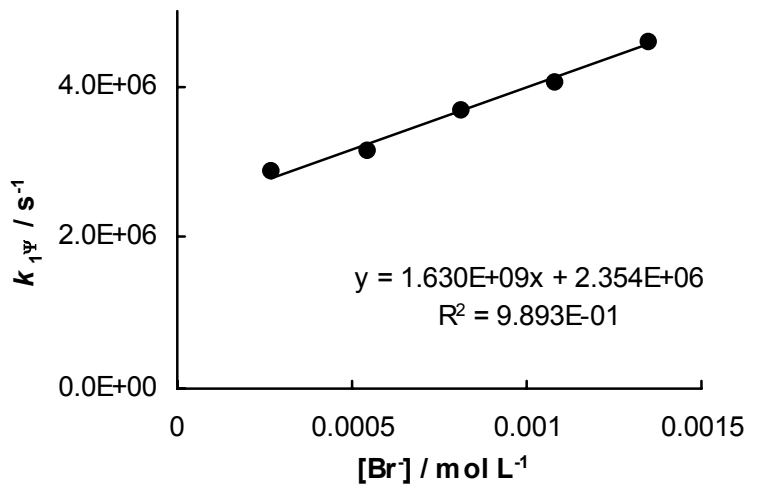


Table S29. (ani) $)_{2} \mathrm{CH}^{+}$from (ani) $)_{2} \mathrm{CHOAc}+\mathrm{Br}^{-}$in $50 \mathrm{E} 50 \mathrm{AN}$ at $20^{\circ} \mathrm{C}$ (Laser flash, detection at 500 $\mathrm{nm}){ }^{a}$

\begin{tabular}{llll}
\hline No. & {$[\text { Precursor }]_{0} / \mathrm{M}$} & {$\left[\mathrm{Br}^{-}\right] / \mathrm{M}$} & $k_{\mathrm{obs}} / \mathrm{s}^{-1}$ \\
\hline F158-1 & $1.59 \times 10^{-4}$ & $1.35 \times 10^{-4}$ & $1.87 \times 10^{6}$ \\
F158-2 & $1.59 \times 10^{-4}$ & $2.70 \times 10^{-4}$ & $3.04 \times 10^{6}$ \\
F158-3 & $1.59 \times 10^{-4}$ & $4.05 \times 10^{-4}$ & $3.46 \times 10^{6}$ \\
F158-4 & $1.59 \times 10^{-4}$ & $5.40 \times 10^{-4}$ & $3.58 \times 10^{6}$ \\
F158-5 & $1.59 \times 10^{-4}$ & $6.75 \times 10^{-4}$ & $4.43 \times 10^{6}$ \\
F158-6 & $1.59 \times 10^{-4}$ & $8.10 \times 10^{-4}$ & $5.09 \times 10^{6}$ \\
\hline
\end{tabular}

${ }^{a} \mathrm{OAc}=$ acetate

$k_{2}=4.32 \times 10^{9} \mathrm{M}^{-1} \mathrm{~s}^{-1}$

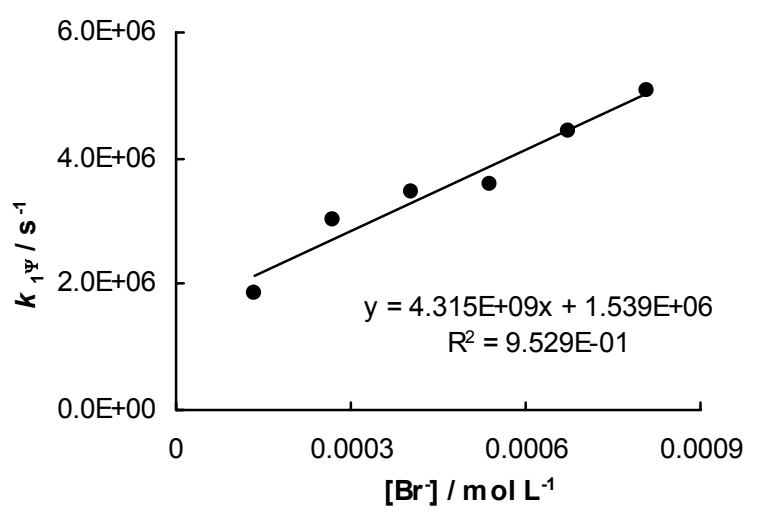


Table S30. (ani) ${ }_{2} \mathrm{CH}^{+}$from (ani) ${ }_{2} \mathrm{CHOAc}+\mathrm{Br}^{-}$in $50 \mathrm{~W} 50 \mathrm{AN}$ at $20^{\circ} \mathrm{C}$ (Laser flash, detection at 500 $\mathrm{nm}){ }^{a}$

\begin{tabular}{llll}
\hline No. & $\begin{array}{l}{[\text { Precursor }]_{0},} \\
\mathrm{~mol} \mathrm{~L}^{-1}\end{array}$ & $\begin{array}{l}{\left[\mathrm{Br}^{-}\right]} \\
\mathrm{mol} \mathrm{L}^{-1}\end{array}$ & $\begin{array}{l}r_{1}+r_{2} \\
\mathrm{~s}^{-1}\end{array}$ \\
\hline F171-1 & $1.59 \times 10^{-4}$ & $1.70 \times 10^{-3}$ & $5.51 \times 10^{5}$ \\
F171-2 & $1.59 \times 10^{-4}$ & $3.40 \times 10^{-3}$ & $7.09 \times 10^{5}$ \\
F171-3 & $1.59 \times 10^{-4}$ & $5.09 \times 10^{-3}$ & $1.07 \times 10^{6}$ \\
F171-4 & $1.59 \times 10^{-4}$ & $6.79 \times 10^{-3}$ & $1.55 \times 10^{6}$ \\
F171-5 & $1.59 \times 10^{-4}$ & $1.02 \times 10^{-2}$ & $2.15 \times 10^{6}$ \\
F171-6 & $1.59 \times 10^{-4}$ & $1.36 \times 10^{-2}$ & $2.65 \times 10^{6}$ \\
\hline
\end{tabular}

${ }^{a} \mathrm{OAc}=$ acetate

$k_{2}=1.86 \times 10^{8} \mathrm{M}^{-1} \mathrm{~s}^{-1}$

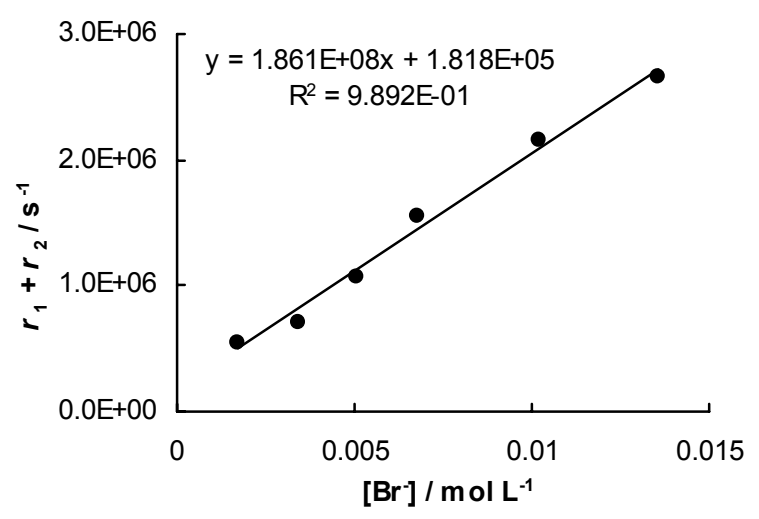


Table S31. (ani) $)_{2} \mathrm{CH}^{+}$from (ani) $)_{2} \mathrm{CHOAc}+\mathrm{Br}^{-}$in $20 \mathrm{~W} 80 \mathrm{AN}$ at $20^{\circ} \mathrm{C}$ (Laser flash, detection at 500 $\mathrm{nm}){ }^{a}$

\begin{tabular}{llll}
\hline No. & {$[\text { Precursor }]_{0} / \mathrm{M}$} & {$\left[\mathrm{Br}^{-}\right] / \mathrm{M}$} & $k_{\mathrm{obs}} / \mathrm{s}^{-1}$ \\
\hline F160-1 & $1.59 \times 10^{-4}$ & $2.70 \times 10^{-4}$ & $3.13 \times 10^{5}$ \\
F160-2 & $1.59 \times 10^{-4}$ & $5.40 \times 10^{-4}$ & $5.29 \times 10^{5}$ \\
F160-3 & $1.59 \times 10^{-4}$ & $8.10 \times 10^{-4}$ & $7.71 \times 10^{5}$ \\
F160-4 & $1.59 \times 10^{-4}$ & $1.08 \times 10^{-3}$ & $8.89 \times 10^{5}$ \\
F160-5 & $1.59 \times 10^{-4}$ & $1.35 \times 10^{-3}$ & $1.08 \times 10^{6}$ \\
\hline
\end{tabular}

${ }^{a} \mathrm{OAc}=$ acetate

$k_{2}=7.02 \times 10^{8} \mathrm{M}^{-1} \mathrm{~s}^{-1}$

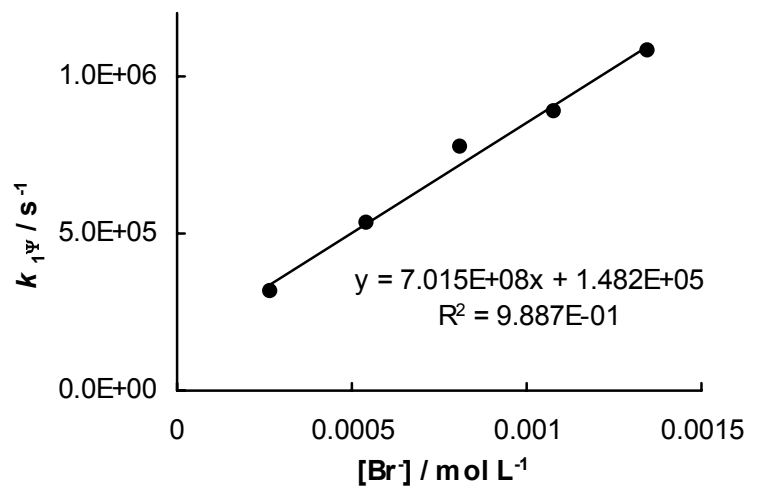


Table S32. (ani)(tol) $\mathrm{CH}^{+}$from (ani)(tol)CHOAc $+\mathrm{Br}^{-}$in $50 \mathrm{E} 50 \mathrm{~W}$ at $20^{\circ} \mathrm{C}$ (Laser flash, detection at 480 nm). ${ }^{a}$

\begin{tabular}{llll}
\hline No. & {$[\text { Precursor }]_{0} / \mathrm{M}$} & {$\left[\mathrm{Br}^{-}\right] / \mathrm{M}$} & $k_{\mathrm{obs}} / \mathrm{s}^{-1}$ \\
\hline F166-1 & $1.51 \times 10^{-4}$ & $9.82 \times 10^{-4}$ & $3.79 \times 10^{6}$ \\
F166-2 & $1.51 \times 10^{-4}$ & $1.96 \times 10^{-3}$ & $5.05 \times 10^{6}$ \\
F166-3 & $1.51 \times 10^{-4}$ & $2.95 \times 10^{-3}$ & $5.71 \times 10^{6}$ \\
F166-4 & $1.51 \times 10^{-4}$ & $3.93 \times 10^{-3}$ & $6.59 \times 10^{6}$ \\
F166-5 & $1.51 \times 10^{-4}$ & $4.91 \times 10^{-3}$ & $7.08 \times 10^{6}$ \\
\hline
\end{tabular}

${ }^{a} \mathrm{OAc}=$ acetate

$k_{2}=8.26 \times 10^{8} \mathrm{M}^{-1} \mathrm{~s}^{-1}$

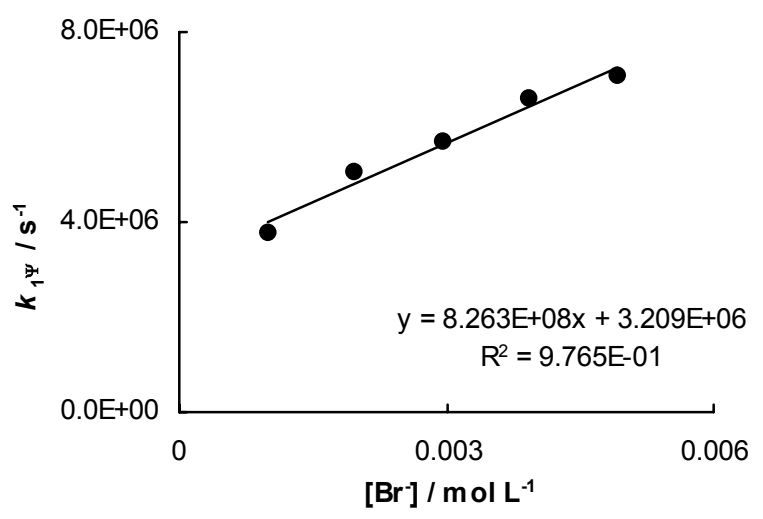


Table S33. (ani) $\mathrm{PhCH}^{+}$from (ani) $\mathrm{PhCHOAr}+\mathrm{Br}^{-}$in $50 \mathrm{E} 50 \mathrm{~W}$ at $20^{\circ} \mathrm{C}$ (Laser flash, detection at 460 $\mathrm{nm}){ }^{a}$

\begin{tabular}{llll}
\hline No. & {$[\text { Precursor }]_{0} / \mathrm{M}$} & {$\left[\mathrm{Br}^{-}\right] / \mathrm{M}$} & $k_{\mathrm{obs}} / \mathrm{s}^{-1}$ \\
\hline F167-1 & $1.36 \times 10^{-4}$ & $6.55 \times 10^{-4}$ & $7.82 \times 10^{6}$ \\
F167-2 & $1.36 \times 10^{-4}$ & $1.31 \times 10^{-3}$ & $8.56 \times 10^{6}$ \\
F167-3 & $1.36 \times 10^{-4}$ & $1.96 \times 10^{-3}$ & $9.16 \times 10^{6}$ \\
F167-4 & $1.36 \times 10^{-4}$ & $2.62 \times 10^{-3}$ & $9.72 \times 10^{6}$ \\
F167-5 & $1.36 \times 10^{-4}$ & $3.27 \times 10^{-3}$ & $1.08 \times 10^{7}$ \\
F167-6 & $1.36 \times 10^{-4}$ & $3.93 \times 10^{-3}$ & $1.18 \times 10^{7}$
\end{tabular}

${ }^{a} \mathrm{OAr}=4$-cyanophenoxide

$k_{2}=1.19 \times 10^{9} \mathrm{M}^{-1} \mathrm{~s}^{-1}$

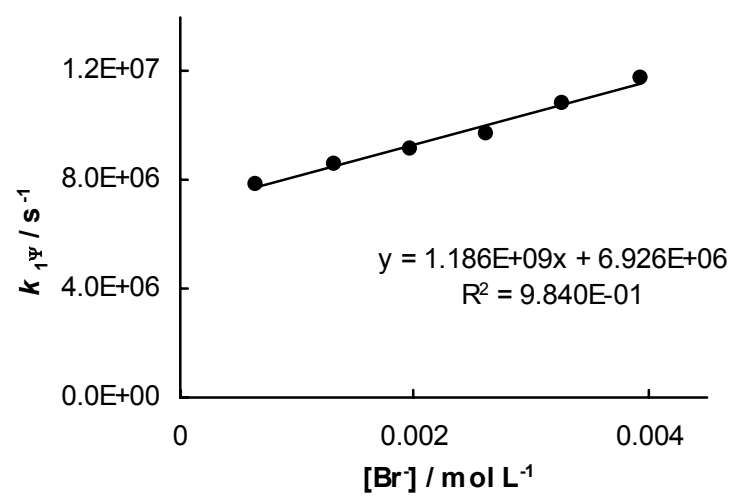


Table S34. (ani) $\mathrm{PhCH}^{+}$from (ani) $\mathrm{PhCHOAr}+\mathrm{Br}^{-}$in TFE at $20^{\circ} \mathrm{C}$ (Laser flash, detection at $460 \mathrm{~nm}$ ). ${ }^{a}$

\begin{tabular}{llll}
\hline No. & {$[\text { Precursor }]_{0} / \mathrm{M}$} & {$\left[\mathrm{Br}^{-}\right] / \mathrm{M}$} & $k_{\text {obs }} / \mathrm{s}^{-1}$ \\
\hline F105-1 & $1.78 \times 10^{-4}$ & $1.94 \times 10^{-3}$ & $6.55 \times 10^{5}$ \\
F105-2 & $1.78 \times 10^{-4}$ & $2.91 \times 10^{-3}$ & $8.98 \times 10^{5}$ \\
F105-3 & $1.78 \times 10^{-4}$ & $3.87 \times 10^{-3}$ & $1.13 \times 10^{6}$ \\
F105-4 & $1.78 \times 10^{-4}$ & $4.84 \times 10^{-3}$ & $1.30 \times 10^{6}$ \\
F105-5 & $1.78 \times 10^{-4}$ & $7.75 \times 10^{-3}$ & $1.73 \times 10^{6}$ \\
F105-6 & $1.78 \times 10^{-4}$ & $9.69 \times 10^{-3}$ & $2.23 \times 10^{6}$
\end{tabular}

${ }^{a} \mathrm{OAr}=4$-cyanophenoxide

$k_{2}=1.92 \times 10^{8} \mathrm{M}^{-1} \mathrm{~s}^{-1}$

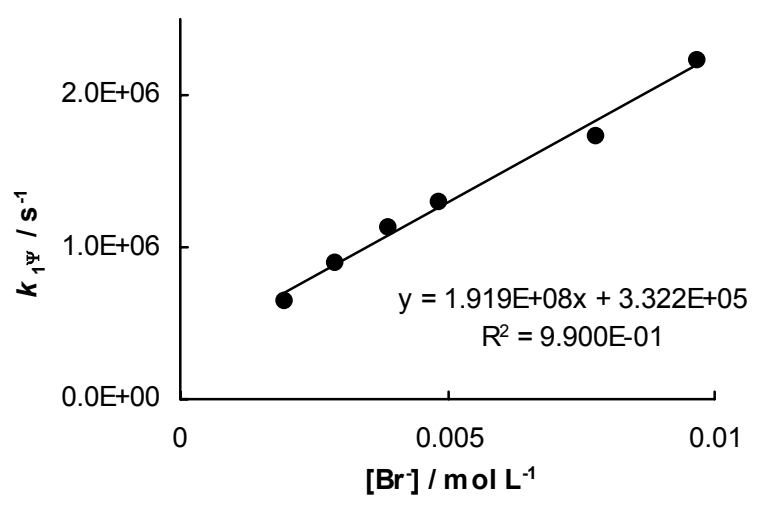


Table S35. (tol) $)_{2} \mathrm{CH}^{+}$from (tol $)_{2} \mathrm{CHOAr}+\mathrm{Br}^{-}$in TFE at $20^{\circ} \mathrm{C}$ (Laser flash, detection at $\left.460 \mathrm{~nm}\right){ }^{a}$

\begin{tabular}{llll}
\hline No. & {$[\text { Precursor }]_{0} / \mathrm{M}$} & {$\left[\mathrm{Br}^{-}\right] / \mathrm{M}$} & $k_{\text {obs }} / \mathrm{s}^{-1}$ \\
\hline F103-1 & $9.44 \times 10^{-5}$ & $1.94 \times 10^{-4}$ & $6.15 \times 10^{5}$ \\
F103-2 & $9.44 \times 10^{-5}$ & $3.87 \times 10^{-4}$ & $1.05 \times 10^{6}$ \\
F103-3 & $9.44 \times 10^{-5}$ & $5.81 \times 10^{-4}$ & $1.46 \times 10^{6}$ \\
F103-4 & $9.44 \times 10^{-5}$ & $7.75 \times 10^{-4}$ & $1.82 \times 10^{6}$ \\
F103-5 & $9.44 \times 10^{-5}$ & $9.69 \times 10^{-4}$ & $2.23 \times 10^{6}$ \\
F85-1 & $9.44 \times 10^{-5}$ & $1.07 \times 10^{-3}$ & $2.42 \times 10^{6}$ \\
F85-2 & $9.44 \times 10^{-5}$ & $1.61 \times 10^{-3}$ & $3.41 \times 10^{6}$ \\
F85-3 & $9.44 \times 10^{-5}$ & $2.14 \times 10^{-3}$ & $4.42 \times 10^{6}$ \\
F85-4 & $9.44 \times 10^{-5}$ & $2.68 \times 10^{-3}$ & $4.98 \times 10^{6}$ \\
F85-5 & $9.44 \times 10^{-5}$ & $3.21 \times 10^{-3}$ & $6.02 \times 10^{6}$ \\
F85-6 & $9.44 \times 10^{-5}$ & $3.75 \times 10^{-3}$ & $7.04 \times 10^{6}$ \\
F85-7 & $9.44 \times 10^{-5}$ & $4.28 \times 10^{-3}$ & $7.17 \times 10^{6}$ \\
F85-8 & $9.44 \times 10^{-5}$ & $5.36 \times 10^{-3}$ & $8.42 \times 10^{6}$ \\
F103-6 & $9.44 \times 10^{-5}$ & $7.75 \times 10^{-3}$ & $1.21 \times 10^{7}$ \\
F103-7 & $9.44 \times 10^{-5}$ & $9.69 \times 10^{-3}$ & $1.43 \times 10^{7}$ \\
\hline OAf & & &
\end{tabular}

${ }^{a} \mathrm{OAr}=4$-cyanophenoxide

$k_{2}=1.76 \times 10^{9} \mathrm{M}^{-1} \mathrm{~s}^{-1}$

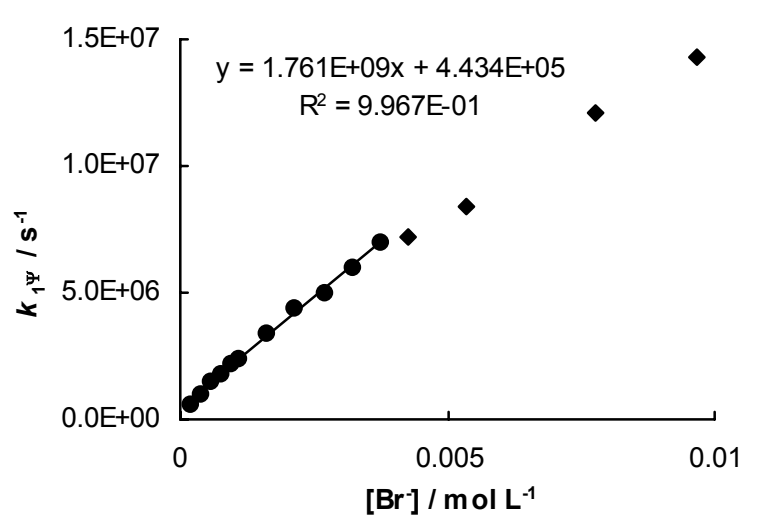


Table S36. (tol) $\mathrm{PhCH}^{+}$from (tol) $\mathrm{PhCHOAr}+\mathrm{Br}^{-}$in $\mathrm{TFE}$ at $20^{\circ} \mathrm{C}$ (Laser flash, detection at $\left.450 \mathrm{~nm}\right){ }^{a}$

\begin{tabular}{llll}
\hline No. & {$[\text { Precursor }]_{0} / \mathrm{M}$} & {$\left[\mathrm{Br}^{-}\right] / \mathrm{M}$} & $k_{\mathrm{obs}} / \mathrm{s}^{-1}$ \\
\hline F107-1 & $1.74 \times 10^{-4}$ & $1.94 \times 10^{-4}$ & $1.28 \times 10^{6}$ \\
F107-2 & $1.74 \times 10^{-4}$ & $3.87 \times 10^{-4}$ & $2.16 \times 10^{6}$ \\
F107-3 & $1.74 \times 10^{-4}$ & $5.81 \times 10^{-4}$ & $2.94 \times 10^{6}$ \\
F107-4 & $1.74 \times 10^{-4}$ & $7.75 \times 10^{-4}$ & $3.51 \times 10^{6}$ \\
F107-5 & $1.74 \times 10^{-4}$ & $9.69 \times 10^{-4}$ & $4.44 \times 10^{6}$ \\
F107-6 & $1.74 \times 10^{-4}$ & $1.36 \times 10^{-3}$ & $5.89 \times 10^{6}$ \\
F107-7 & $1.74 \times 10^{-4}$ & $1.94 \times 10^{-3}$ & $8.13 \times 10^{6}$ \\
\hline
\end{tabular}

${ }^{a} \mathrm{OAr}=4$-cyanophenoxide

$k_{2}=3.89 \times 10^{9} \mathrm{M}^{-1} \mathrm{~s}^{-1}$

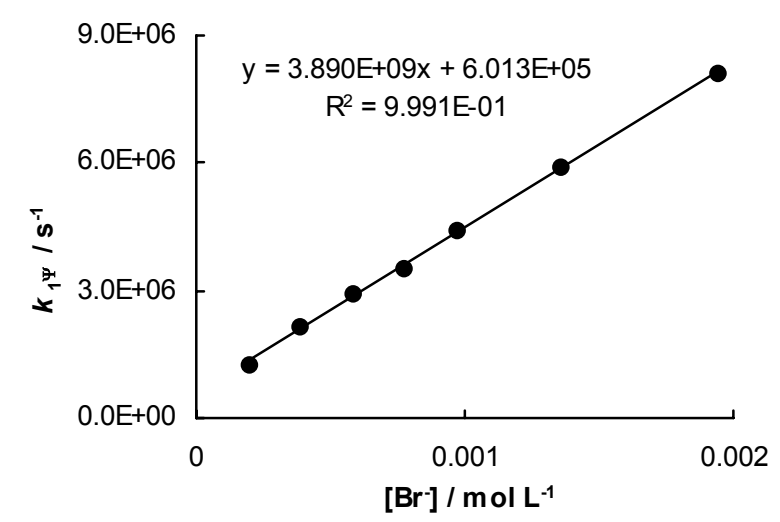


Table S37. $\mathrm{Ph}_{2} \mathrm{CH}^{+}$from $\mathrm{Ph}_{2} \mathrm{CHOAr}+\mathrm{Br}^{-}$in HFIP at $20^{\circ} \mathrm{C}$ (Laser flash, detection at $\left.440 \mathrm{~nm}\right){ }^{a}$

\begin{tabular}{llll}
\hline No. & {$[\text { Precursor }]_{0} / \mathrm{M}$} & {$\left[\mathrm{Br}^{-}\right] / \mathrm{M}$} & $k_{\mathrm{obs}} / \mathrm{s}^{-1}$ \\
\hline F140-1 & $2.23 \times 10^{-4}$ & $2.30 \times 10^{-4}$ & $8.27 \times 10^{5}$ \\
F140-2 & $2.23 \times 10^{-4}$ & $4.59 \times 10^{-4}$ & $1.62 \times 10^{6}$ \\
F140-3 & $2.23 \times 10^{-4}$ & $6.89 \times 10^{-4}$ & $2.16 \times 10^{6}$ \\
F140-4 & $2.23 \times 10^{-4}$ & $9.18 \times 10^{-4}$ & $2.46 \times 10^{6}$ \\
F140-5 & $2.23 \times 10^{-4}$ & $1.15 \times 10^{-3}$ & $3.04 \times 10^{6}$ \\
F140-6 & $2.23 \times 10^{-4}$ & $2.07 \times 10^{-3}$ & $5.66 \times 10^{6}$ \\
F140-7 & $2.23 \times 10^{-4}$ & $3.90 \times 10^{-3}$ & $7.43 \times 10^{6}$ \\
F140-8 & $2.23 \times 10^{-4}$ & $5.97 \times 10^{-3}$ & $1.02 \times 10^{7}$ \\
F140-9 & $2.23 \times 10^{-4}$ & $8.04 \times 10^{-3}$ & $1.28 \times 10^{7}$ \\
F140-10 & $2.23 \times 10^{-4}$ & $9.87 \times 10^{-3}$ & $1.36 \times 10^{7}$ \\
F140-11 & $2.23 \times 10^{-4}$ & $1.15 \times 10^{-2}$ & $1.55 \times 10^{7}$ \\
\hline
\end{tabular}

${ }^{a} \mathrm{OAr}=4$-cyanophenoxide

$k_{2}=2.55 \times 10^{9} \mathrm{M}^{-1} \mathrm{~s}^{-1}$

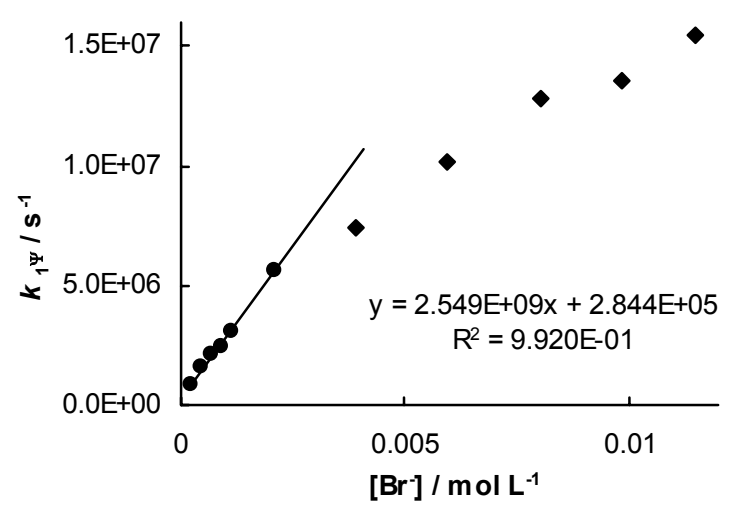


Table S38. Rate constants for the reaction of (fur) $)_{2} \mathrm{CHCl}$ with $n \mathrm{Bu}_{4} \mathrm{~N}^{+} \mathrm{Br}$ in $\mathrm{AN}$ by Laser flash $(\lambda=520$ $\left.\mathrm{nm}, 20^{\circ} \mathrm{C}\right)$.

\begin{tabular}{cccc}
\hline Nr. & {$[\mathrm{E}]_{0} / \mathrm{M}$} & {$[\mathrm{Nu}]_{0} / \mathrm{M}$} & $k_{\psi} / \mathrm{s}^{-1}$ \\
\hline 15030208 & $3.59 \times 10^{-5}$ & $2.75 \times 10^{-4}$ & $4.30 \times 10^{6}$ \\
15030206 & $3.59 \times 10^{-5}$ & $5.50 \times 10^{-4}$ & $6.84 \times 10^{6}$ \\
15030209 & $3.59 \times 10^{-5}$ & $8.25 \times 10^{-4}$ & $1.02 \times 10^{7}$ \\
15030207 & $3.59 \times 10^{-5}$ & $1.10 \times 10^{-3}$ & $1.36 \times 10^{7}$ \\
\hline
\end{tabular}

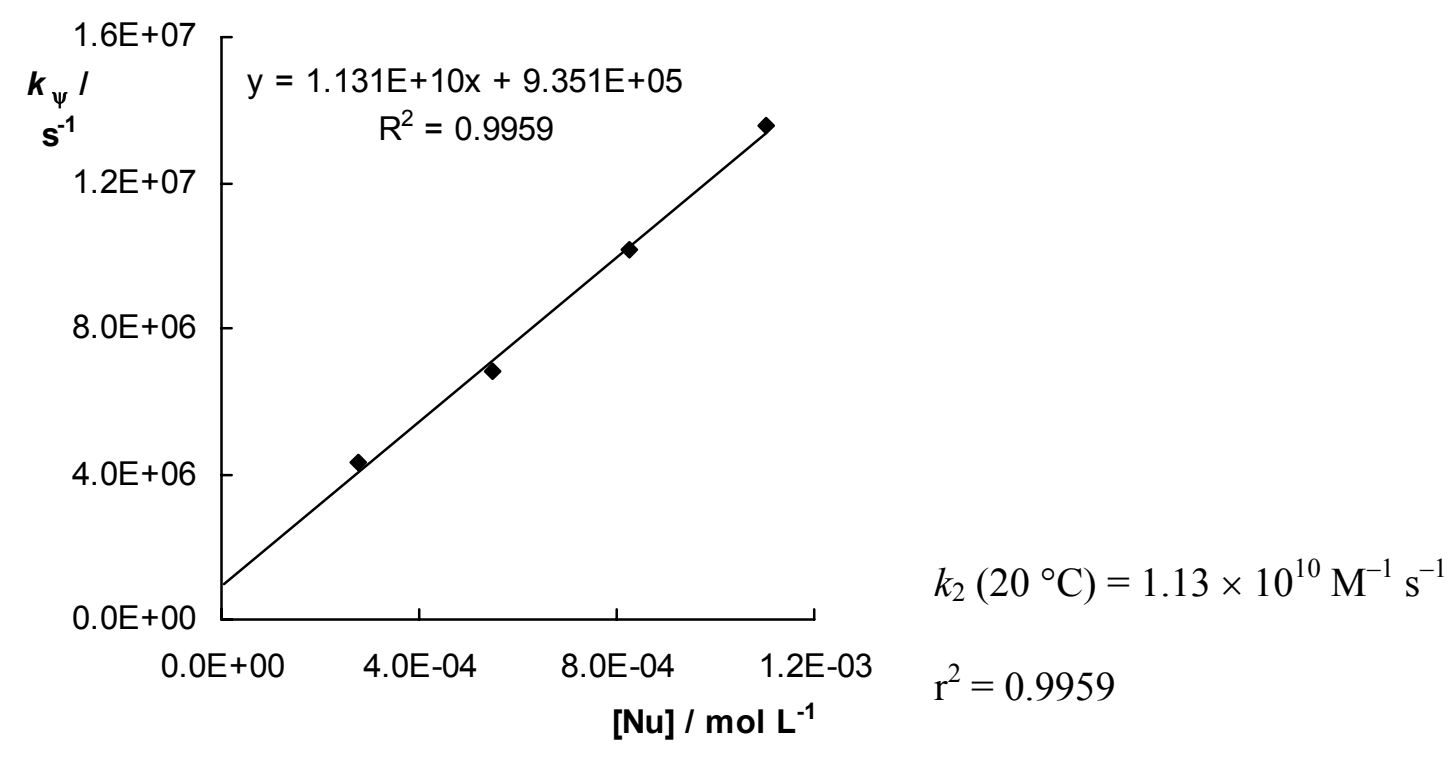


Table S39. Rate constants for the reaction of (pfa) ${ }_{2} \mathrm{CH}^{+} \mathrm{BF}_{4}^{-}$and additional 1.12 eq of $p-\mathrm{CN}_{-} \mathrm{C}_{6} \mathrm{H}_{4} \mathrm{O}^{-}$ $\mathrm{Na}^{+}$with $n \mathrm{Bu}_{4} \mathrm{~N}^{+} \mathrm{Br}^{-}$in AN by Laser flash $\left(\lambda=592 \mathrm{~nm}, 20^{\circ} \mathrm{C}\right)$.

\begin{tabular}{ccccc}
\hline $\mathrm{Nr}$. & {$[\mathrm{E}]_{0} / \mathrm{M}$} & {$\left[p-\mathrm{CN}-\mathrm{PhO}^{-} \mathrm{Na}^{+}\right] / \mathrm{M}$} & {$[\mathrm{Nu}]_{0} / \mathrm{M}$} & $k_{\psi} / \mathrm{s}^{-1}$ \\
\hline 09040220 & $4.78 \times 10^{-5}$ & $5.34 \times 10^{-5}$ & $1.04 \times 10^{-3}$ & $2.23 \times 10^{6}$ \\
09040221 & $4.78 \times 10^{-5}$ & $5.34 \times 10^{-5}$ & $2.07 \times 10^{-3}$ & $3.99 \times 10^{6}$ \\
09040222 & $4.78 \times 10^{-5}$ & $5.34 \times 10^{-5}$ & $4.15 \times 10^{-3}$ & $6.73 \times 10^{6}$ \\
09040223 & $4.78 \times 10^{-5}$ & $5.34 \times 10^{-5}$ & $5.19 \times 10^{-3}$ & $8.07 \times 10^{6}$ \\
\hline
\end{tabular}

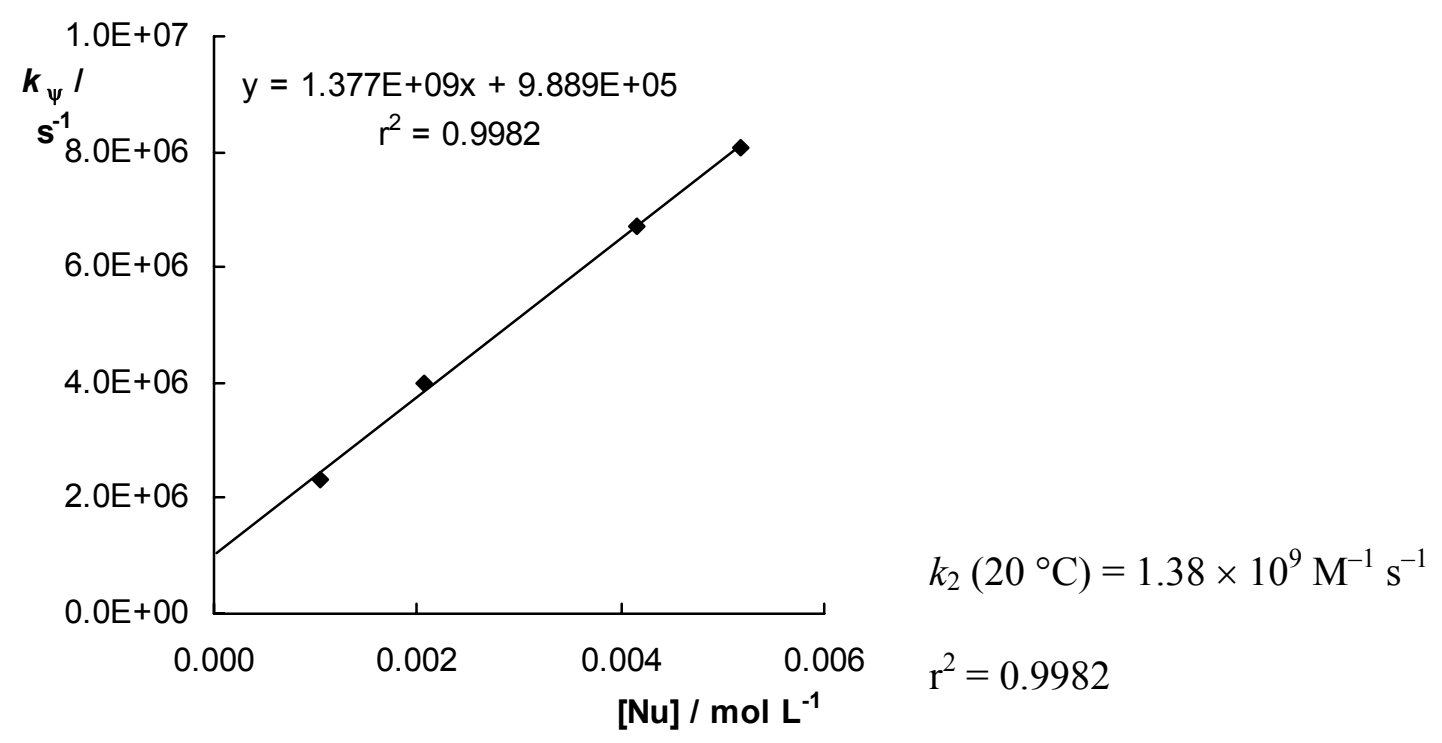


Table S40. Rate constants for the reaction of (pfa) ${ }_{2} \mathrm{CH}^{+} \mathrm{BF}_{4}^{-}$with $n \mathrm{Bu}_{4} \mathrm{~N}^{+} \mathrm{Br}^{-}{ }^{-[a]}$ in $\mathrm{AN}$ by Laser flash $(\lambda$ $=592 \mathrm{~nm}, 20^{\circ} \mathrm{C}$ ).

\begin{tabular}{cccc}
\hline Nr. & {$[\mathrm{E}]_{0} / \mathrm{M}$} & {$[\mathrm{Nu}]_{0} / \mathrm{M}$} & $k_{\psi} / \mathrm{s}^{-1}$ \\
\hline 02050209 & $2.88 \times 10^{-5}$ & $4.98 \times 10^{-4}$ & $1.74 \times 10^{6}$ \\
02050201 & $2.88 \times 10^{-5}$ & $9.96 \times 10^{-4}$ & $2.50 \times 10^{6}$ \\
02050202 & $2.88 \times 10^{-5}$ & $1.49 \times 10^{-3}$ & $3.21 \times 10^{6}$ \\
02050204 & $2.88 \times 10^{-5}$ & $1.49 \times 10^{-3}$ & $3.32 \times 10^{6}$ \\
02050203 & $2.88 \times 10^{-5}$ & $1.99 \times 10^{-3}$ & $3.81 \times 10^{6}$ \\
02050208 & $2.88 \times 10^{-5}$ & $2.49 \times 10^{-3}$ & $4.55 \times 10^{6}$
\end{tabular}

${ }^{a}$ Solutions still blue prior to the laser-pulse.

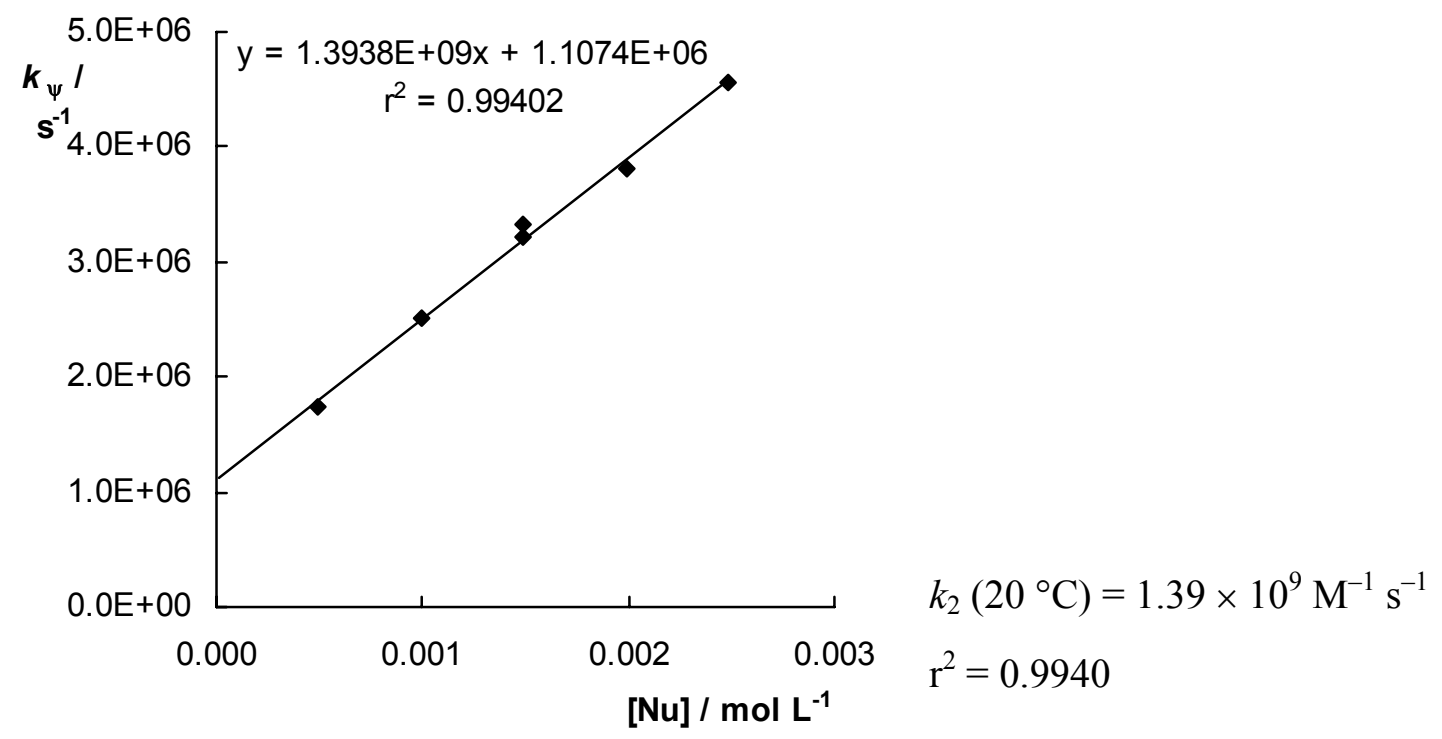




\section{References}

(1) (a) McClelland, R. A.; Kanagasabapathy, V. M.; Banait, N. S.; Steenken, S. J. Am. Chem. Soc. 1989, 111, 3966-3972. (b) Pham, T. V.; McClelland, R. A. Can. J. Chem. 2001, 79, 1887-1897.

(2) Chiang, Y.; Kresge, A. J.; Zhu, Y.J. Am. Chem. Soc. 2002, 124, 6349-6356.

(3) Hammett, L. P. Physical Organic Chemistry: Reaction Rates, Equilibria, and Mechanisms, 2nd ed.; McGraw-Hill: New York, 1970.

(4) Bentley, T. W.; Llewellyn, G. Prog. Phys. Org. Chem. 1990, 17, 121-159. 